LBNL-42409

\title{
Experimental Validation of the Wavefield Transform
}

\author{
Kaushik Kunal Das \\ M.S. Thesis \\ Earth Sciences Division \\ Ernest Orlando Lawrence Berkeley National Laboratory \\ University of California \\ Berkeley, CA 94720
}

December 1996

This work was supported by the Director, Office of Science, Office of Basic Energy Sciences, Engineering and Geosciences Division, of the U.S. Department of Energy under Contract No. DE-AC03-76SF00098. 


\section{DISCLAIMER}

This report was prepared as an account of work sponsored by an agency of the United States Government. Neither the United States Government nor any agency thereof, nor any of their employees, make any warranty, express or implied, or assumes any legal liability or responsibility for the accuracy, completeness, or usefulness of any information, apparatus, product, or process disclosed, or represents that its use would not infringe privately owned rights. Reference herein to any specific commercial product, process, or service by trade name, trademark, manufacturer, or otherwise does not necessarily constitute or imply its endorsement, recommendation, or favoring by the United States Government or any agency thereof. The views and opinions of authors expressed herein do not necessarily state or reflect those of the United States Government or any agency thereof. 


\section{DISCLAIMER}

Portions of this document may be illegible in electronic image products. Images are produced from the best available original document. 
LBNL-42409

\title{
Experimental Validation of the Wavefield Transform
}

\author{
by \\ Kaushik Kunal Das \\ B. Tech. (Institute of Technology of the Banaras Hindu University) 1993 \\ A thesis submitted in partial satisfaction of the requirements for the degree of \\ Master of Science \\ in \\ Engineering-Materials Science \\ and Mineral Engineering \\ In the \\ GRADUATE DIVISION \\ of the \\ UNIVERSITY of CALIFORNIA at BERKELEY
}

Committee in charge:

Professor Alex Becker, Chair

Professor H. Frank Morrison

Professor James Rector

Fall 1996 


\title{
Experimental Validation of the Wavefield Transform
}

\author{
Copyright () 1996
}

by

Kaushik Kunal Das

The U.S. Department of Energy has the right to use this document for any purpose whatsoever including the right to reproduce all or any part thereof. 


\author{
Abstract \\ Experimental Validation of the Wavefield Transform \\ by \\ Kaushik Kunal Das \\ Master of Science in Engineering \\ University of California at Berkeley \\ Professor Alex Becker, Chair
}

The advent of sophisticated inversion techniques for interpreting subsurface electromagnetic data brought with it stringent demands for improvements in the accuracy and fidelity of measurements. In particular the wavefield transformation technique makes it possible to map the interwell distribution of electrical conductivity if the data is noisefree over a large bandwidth. In that case it becomes possible to form a tomographic image of the subsurface section bounded by two vertical boreholes. In this technique the diffusive low frequency EM field is numerically transformed to a mathematically defined space where it constitutes a wavefield. The resultant pulse has a velocity which is dependent on the conductivity of the medium. Thus, an image of the subsurface distribution of electrical conductivity can be constructed using a non-linear ray tracing technique normally reserved for seismic velocity tomography (Lee and Xie, 1993).

A fully computerized laboratory scale time domain data acquisition system has been designed and used to simulate subsurface crosswell and borehole-to-surface experiments 
in a horizontally layered earth model. The model is made of two cylindrical blocks of graphite with an electrical conductivity of $9.4 \times 10^{4} \mathrm{~S} / \mathrm{m}$ and $1.4 \mathrm{~m}$ in diameter. Steel sheets with a conductivity of $1.39 \times 10^{6} \mathrm{~S} / \mathrm{m}$ and $1.2 \mathrm{~mm}$ thick are also used. This model has been linearly scaled down from field dimensions by a factor of 1000 . The conductivity has been scaled up by a factor of $10^{6}$. The time scale is unity.

The acquired laboratory scale model data were successfully transformed to the wavefield domain. Thus the practical feasibility of the wavefield transform technique was established. In the course of this process it was observed that the system bandwidth is a crucial parameter affecting data fidelity. 
Acknowledgments:

I wish to convey my sincere thanks to my advisor Professor Alex Becker whose guidance and motivation has been invaluable. Dr. K. H. Lee and Dr. Ganquan Xie of Lawrence Berkeley National Laboratories provided constant support and encouragement. I would also like to thank the faculty and students of the Engineering Geoscience Group for much helpful discussion. Funding for the project was provided by the Office of Basic Energy Sciences, Engineering and Geosciences Division of the U.S. Department of Energy under contract no. DE-AC03-76SF00098. 


\section{TABLE OF CONTENTS}

1. INTRODUCTION 1

1.1 Overview 1

1.2 Background 3

1.3 Objectives 4

2. THEORY 5

2.1 The Wavefield Transform 5

2.2 Scaling Relations for the Laboratory Model 8

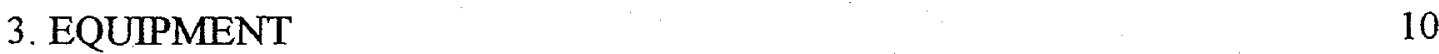

3.1 Description 10

$\begin{array}{ll}3.2 \text { Signal processing } & 13\end{array}$

3.3 Signal fidelity 14

3.3.1 Bandwidth 14

3.3.2 External Noise $\quad 17$

3.3.3 Data Quality 18

$\begin{array}{lr}\text { 3.3.4 Sensor Orientation } & 19\end{array}$

$\begin{array}{lll}\text { 4.RESULTS } & 20\end{array}$

4.1 Cross-borehole experiment in uniform medium 21

4.2 Cross-borehole experiment across highly conducting thin sheet 23

4.3 Cross-borehole experiment across variable thickness conducting sheet 24

4.4 Surface-to-borehole experiment 26 
6. REFERENCES

Appendix: The users manual for the wavefield transform program 


\section{LIST OF FIGURES}

3.I Block diagram of the laboratory setup

3.2 Effect of time-windowing on data

3.3 Receiver Circuit

3.4 Effect of system bandwidth in time domain

3.5 Effect of system bandwidth in wave domain

3.6 (a) Effect of system bandwidth in frequency domain - Amplitude

(b) Effect of system bandwidth in frequency domain - Phase

3.7 Reciprocity test in cross-borehole configuration

3.8 Comparison between theory and experiment

3.9 (a) Effect of rotation of receiver coil in time domain

3.9 (b) Effect of rotation of receiver coil in wave domain

4.1 (a) Cross-borehole dataset - system configuration

(b) Cross-borehole dataset - Time Domain

(c) Cross-borehole dataset - Wave Domain

4.2 (a) Cross-borehole dataset across highly conducting thin sheet - system configuration

(b) Cross-borehole dataset across highly conducting thin sheet - Time Domain

(c) Cross-borehole dataset across highly conducting thin sheet - Wave Domain

4.3 (a) Cross-borehole dataset across variable thickness steel sheet - system configuration

(b) Cross-borehole dataset across variable thickness steel sheet - Time Domain 
(c) Cross-borehole dataset across variable thickness steel sheet - Wave Domain

4.4 (a) Borehole-to-surface dataset - system configuration

(b) Borehole-to-surface dataset - Time Domain

(c) Borehole-to-surface dataset - Wave Domain 


\section{LIST OF TABLES}

3. 1 Laboratory model parameters

4.1 Direct wavefield arrivals for experiment 4.1

4.2 Direct wavefield arrivals for experiment 4.2

4.3 Direct wavefield arrivals for experiment 4.3

4.4 Direct wavefield arrivals for experiment 4.4 


\section{INTRODUCTION}

\subsection{Overview}

The surface of the earth is opaque to human eyes as optical frequency electromagnetic waves cannot penetrate it. The goal of subsurface imaging is to extend the limits of the human eye by using a low frequency forcefield and tracking its passage as the eye does with optical rays. Propagation of any field through a medium is dependent on its properties. This means that after the field has passed through that medium it contains information about the distribution of those properties. The recovery of that information enables us to form an image of that distribution.

Here we consider the electromagnetic (EM) forcefield. The property of the medium in that case becomes electrical conductivity. Conductivity images can be used to map groundwater and contaminant plumes, characterize petroleum and geothermal reservoirs, monitor subsurface processes (waste remediation and emplacement, enhanced petroleum recovery, reservoir production, long term changes in vadose zone saturation), resource exploration, and general geological mapping of the crust of the earth. To be more specific, low frequency EM fields $(<1 \mathrm{MHz})$ need to be used for the depths of interest in such cases which range from a few meters to 10 or $20 \mathrm{~km}$. At low frequencies however, the prevalent values of electrical conductivity in geological materials preclude the dispersionless propagation of electromagnetic energy in the form of waves such as 
those that are used for radar. Instead, the transmitted fields diffuse through the subsurface so that conventional wave propagation concepts like rays and traveltimes are inoperative here. As a further consequence we find that the data processing and inverse techniques such as ray tracing or migration that are so useful for interpreting seismic wave data cannot be of direct help in this case.

One way to obtain an image of the subsurface from low frequency EM fields is to mathematically transform the diffused arrival waveforms into pseudo-sonic wavelets. This procedure renders the low frequency EM field as easy to visualize as high frequency EM fields or seismic waves. It also becomes possible to exploit many of the numerous, highly developed, wavefield processing methods used to interpret seismic wave data. The desired result can be achieved by the application of a wavefield transformation to the observed EM field which transforms it from a physical space-time to a mathematically defined space-time where a time-like variable " $q$ " replaces time. The spatial coordinates are unaffected by the transform. The transformation procedure however requires very high quality EM data in the time domain. Our task was the design and implementation of a laboratory scale time domain EM data acquisition system intended to demonstrate that the transformation can be done under practical working conditions. The subsequent pages contain a detailed description of the experiments and the equipment used to perform them. 


\subsection{Background}

The theoretical basis for our work is derived from the most generalized form of the wavefield transform that was presented by Lee et al. (1989). It is used to relate the time-dependent diffusive electromagnetic field to a unique wavefield in the domain of a time-like variable named "q". The transformed diffusive EM field satisfies a wave equation where the velocity of the wave is inversely proportional to the square root of the electrical conductivity. Mapping the velocity, or the slowness, of the wavefield then gives the sought conductivity map. Thus, experimental data collected in the time domain may be transformed to wavefields and a tomographic technique can be used to image the electrical conductivity distribution. The practicality of this technique was successfully demonstrated by Lee and Xie (1993) using numerical model data but previous attempts to use field data (Wilson et al, 1993) have encountered severe shortcomings in the fidelity of the results.

An analysis of the transformation problem (Lee, 1989) showed that construction of a wavefield with reasonable waveform resolution requires about four decades of time- or frequency-domain data with a maximum allowable noise of about three percent. Nevertheless, useful information, such as traveltime, is retained in the transformed wavefield even if the time window is substantially reduced. Thus, a numerical algorithm based on singular value decomposition (Lee and Xie, 1993) can be used to get reasonably accurate traveltime information from only 1.5 decades of time-domain data as long as the 
noise is kept under a three percent limit. Most of the recent work on the wavefield transform execution has been focused on the improvement in efficiency of the wavefield transform methods and relaxation in data requirements (Gershenson, 1993; Lee et al., 1994; Slob et al, 1995; Wilson et al., 1995).

\subsection{Objectives}

Analysis of EM data using the wavefield transform has traced the evolutionary path of any new technique, from its genesis as an idea to its demonstration with the aid of numerically generated data. The next logical stage is a test based on experimental data and the principal objective of this project was to acquire such data to establish the validity of the wavefield transform technique. Since the process of data transformation from the time domain to the q-domain is numerically ill-posed, the data have to be extensive as well as noise-free. Our aim was to meet the Lee and Xie (1993) criteria in an experimental environment.

To do this we constructed a laboratory scale model to simulate a rudimentary petroleum reservoir. To keep pace with the theory we chose a cross-well configuration for the experiments. In order to meet the stringent data quality requirements we had to implement a fairly advanced data acquisition system whose electrical parameters had to be

closely controlled. Once established, the system parameters could be profitably transferred to the design of full scale system. 


\section{THEORY}

\subsection{The Wavefield Transformation}

If we neglect the displacement currents, the ambient magnetic field is described by a second order partial differential equation of the form that governs any diffusion process (Ward and Hohmann, 1989) :

$\nabla^{2} \vec{H}(\vec{r}, t)-\mu \sigma(\vec{r}) \frac{\partial}{\partial t} \vec{H}(\vec{r}, t)=\vec{S}(\vec{r}, t)$

where all quantities are in SI units and,

$\vec{H}=$ magnetic field,

$\vec{r}=$ position vector

$\mathrm{t}$ = time,

$\mu=$ magnetic permeability,

$\sigma=$ electrical conductivity,

$\vec{S}(\vec{r}, t)=-\vec{\nabla} \times \overrightarrow{J_{S}}(\vec{r}, t)$ is the source term, 


\section{$\overrightarrow{J_{S}}=$ impressed current.}

Now we go to the q-domain and create functions $\vec{U}(\vec{r}, q)$ and $\vec{F}(\vec{r}, q)$ such that

$\nabla^{2} \vec{U}(\vec{r}, q)-\mu \sigma(\vec{r}) \frac{{ }^{2} \partial}{\partial q^{2}} \vec{U}(\vec{r}, q)=\vec{F}(\vec{r}, q)$

It can then be shown, as was done by Lee et al. (1989) that the magnetic field $\mathrm{H}$ and the wavefield $U$ are uniquely related by

$$
\vec{H}(\vec{r}, t)=\frac{1}{2 \sqrt{\pi t^{3}}} \int_{0}^{\infty} q \exp \left(-\frac{q^{2}}{4 t}\right) \vec{U}(\vec{r}, q) d q
$$

This transform operates between $\mathrm{t}$ and $\mathrm{q}$ exclusively and is independent of the space variable $\vec{r}$. Making a comparison between equations $(2.1)$ and $(2.2)$ it is clear that the independent variable $q$ has the dimensions of the square root of time. Consequently the function $\vec{U}(\vec{r}, q)$ behaves as if it were propagating with a velocity of $(\mu \sigma)^{-1 / 2}$ in $m / \sqrt{s}$. The source functions $S(t)$ and $F(q)$ are also related by the same transform.

The inverse transform, from $\vec{H}(\overrightarrow{r, t)}$ to $\vec{U}(\vec{r}, t)$, is an ill-posed problem that is highly sensitive to noise in the data. Nevertheless it can be formulated as a deconvolution (Lee, 1989) and carried out using a stochastic approach. The construction of the wavefield with reasonable resolution by this method requires approximately four decades of time domain or frequency domain data with a maximum allowable noise in the power spectrum of 
about three percent. However, if a singular value decomposition method is used after regularizing the transformation matrix (Lee and Xie, 1993) then only 1.5 decades of data are enough to retain useful information such as the traveltime.

To take advantage of the singular value decomposition method we proceed as follows. First, equation (2.3) is written in a discreet form by using the trapezoidal integral formula and the collocation method -

$8 \sqrt{\pi t_{i}^{3}} H_{i}=q_{1} e^{-\frac{q_{1}^{2}}{4 t_{i}}}\left(q_{2}-q_{1}\right) U_{1}+2 \sum_{j=2}^{m-1} q_{j} e^{-\frac{q_{j}^{2}}{4 t_{i}}}\left(q_{j+1}-q_{j-1}\right) U_{j}+q_{m} e^{-\frac{q_{m}^{2}}{4 t_{i}}}\left(q_{m}-q_{m-1}\right) U_{m}$

for $i=1,2, \ldots, n(n>=m)$,

where we discretise the $\mathrm{H}$ and $\mathrm{U}$ functions to form

$H_{i}=H\left(t_{i}\right)$ and $U_{j}=U\left(q_{j}\right)$,

and

$q_{j}=(j-1) \Delta q$

$t_{i}=\frac{(i-1)\left(t_{b}-t_{a}\right)}{n-1}$

Here $\Delta \mathrm{q}$ is the increment in $\mathrm{q}$ for the wavefield and $\left\{t_{a}, t_{b}\right\}$ is the time window occupied by the time-domain data. Equation (2.4) can be written in the matrix form as $\mathrm{AU}=\mathrm{H}$.

The matrix A may be decomposed for singular value decomposition as $A=X \Lambda V^{T}$

where $\Lambda$ is diagonal, consisting of singular values, and matrices $X$ and $V$ are orthonormal. 
The diagonal matrix is numerically singular and requires regularization. Then the solution for $U$ can be written as

$U=V\left(\Lambda^{2}+\alpha\right)^{-1} \Lambda X^{T} H$

where alpha is a regularizing parameter and $I$ is an identity matrix of order $m$. The regularizing parameter alpha effectively controls the threshold singular value in equation (2.6). Lee and Xie (1993) have used a quasi-optimality method to choose it. Practically its optimal value has to be determined for any particular data set and depends on the noise level in the data. When the data are noisy a larger value of alpha has to be employed. This results in a smoother wavefield solution at the cost of resolution. The size of the increment in q sets the upper limit of resolution in wavefield domain.

\subsection{Scaling relations for the laboratory model}

The laboratory model was constructed to represent a realistic field situation where the ground resistivity is $0.1 \mathrm{ohm}-\mathrm{m}$. and the transmitter-receiver separation is $50 \mathrm{~m}$. The time scale is unity so that it is possible to use field equipment on the scale model. Distance scaling is compensated entirely by the high conductivity of the model material.

Following Grant and West (1965), the relation between field and model parameters for a quasi-static field can be expressed as 


$$
\begin{aligned}
& \frac{\mu_{f} \omega_{f} \sigma_{f} l_{f}^{2}}{\mu_{m} \omega_{m} \sigma_{m} l_{m}^{2}}=1 \text { or, } \\
& \frac{\mu_{f} t_{m} \sigma_{f} l_{f}^{2}}{\mu_{m} t_{f} \sigma_{m} l_{m}^{2}}=1,
\end{aligned}
$$

where the subscript " $\mathrm{f}$ " stands for a field dimension and the subscript " $m$ " stands for a model dimension and -

$\mu=$ magnetic permeability,

$\omega=$ angular frequency,

$\sigma=$ electrical conductivity

1 = length scale, and

$\mathrm{t}=$ time scale.

If we want the time scale to remain unchanged, the scaling relation becomes $\sigma_{m} l_{m}^{2}=\sigma_{f} l_{f}^{2}$ since the magnetic permeability scale is considered unity. This results in a linear scale of $1: 1000$ and a conductivity scale of $10^{6}: 1$. In our case we used graphite with a conductivity of $94,000 \mathrm{~S} / \mathrm{m}$ to represent a reservoir conductivity of $94 \mathrm{mS} / \mathrm{m}$. similar analysis carried out in the wavefield domain leads to the following relation -

$$
\frac{\sigma_{m} l_{m}^{2}}{q_{m}^{2}}=\frac{\sigma_{f} l_{f}^{2}}{q_{f}^{2}}
$$

This means that the q-scale factor is also unity. 


\section{EQUIPMENT}

\subsection{Description}

The experiment was designed to simulate field data acquisition procedures in the laboratory. A detailed description of the equipment was given by Bentley (1993), so that only the main characteristics of the system are listed here. The model was made up of a number of superposed flat lying cylindrical layers. The diameter of the model structure was $1.4 \mathrm{~m}$. Two vertical $1 \mathrm{~cm}$ diameter holes spaced by a distance of $5 \mathrm{~cm}$ were drilled through the structure.

TABLE 3.1 LABORATORY MODEL PARAMETERS

\section{MODEL}

FIELD

\section{STRUCTURE}

Top layer

- Material

Graphite

Shale

- Conductivity

$94,000 \mathrm{~S} / \mathrm{m}$

$0.094 \mathrm{~S} / \mathrm{m}$

- Thickness

$0.15 \mathrm{~m}$ (top)

$150 \mathrm{~m}$ 


\section{LABORATORY}

FIELD

(continued)

Middle layer

- Material

Stainless Steel

Saline Sandstone

- Conductivity

$1,390,000 \mathrm{~S} / \mathrm{m}$

$1.39 \mathrm{~S} / \mathrm{m}$

- Thickness

variable

$(1.2,2.4$ or $3.6 \mathrm{~mm})$

$1.2,2.4$ or $3.6 \mathrm{~m}$

Bottom layer

-.Material

Graphite

Shale

-.Conductivity

$94,000 \mathrm{~S} / \mathrm{m}$

$0.094 \mathrm{~S} / \mathrm{m}$

- Thickness

$0.30 \mathrm{~m}$

$300 \mathrm{~m}$

SYSTEM PARAMETERS

Bandwidth

$0-0.5 \mathrm{MHz}$

Recording Interval

1-1024 microseconds

Sampling Rate

$1 \mathrm{MHz}$

Dynamic Range

$72 \mathrm{~dB}$ 
TRANSMITTER

Type

Effective Area

Driver

Current

Primary Field Function

Duty Cycle

Ramp Time

RECEIVER

Type

Signal measured

Effective Area
Air Cored Dipole

$200 \mathrm{sq} . \mathrm{cm}$.

GEONICS PROTEM-47

$0.3 \mathrm{~A}$

Boxcar Pulse of alternating polarity

$50 \%$

$<2$ microseconds
Air Cored Dipole

$\mathrm{dB} / \mathrm{dt}$

$200 \mathrm{sq} . \mathrm{cm}$.

A block diagram of the system is given in figure 3.1. A Geonics PROTEM 47 EM set is used to generate the transmitter field. The receiver of the set is used to trigger the transmitter unit which drives the transmitter coil. The current pulse is also used to trigger the analog-to-digital converter which digitizes the signal from the receiver after it has been amplified by a low-noise pre-amplifier and sends it to a desktop computer. The complete data acquisition procedure is computer controlled. 


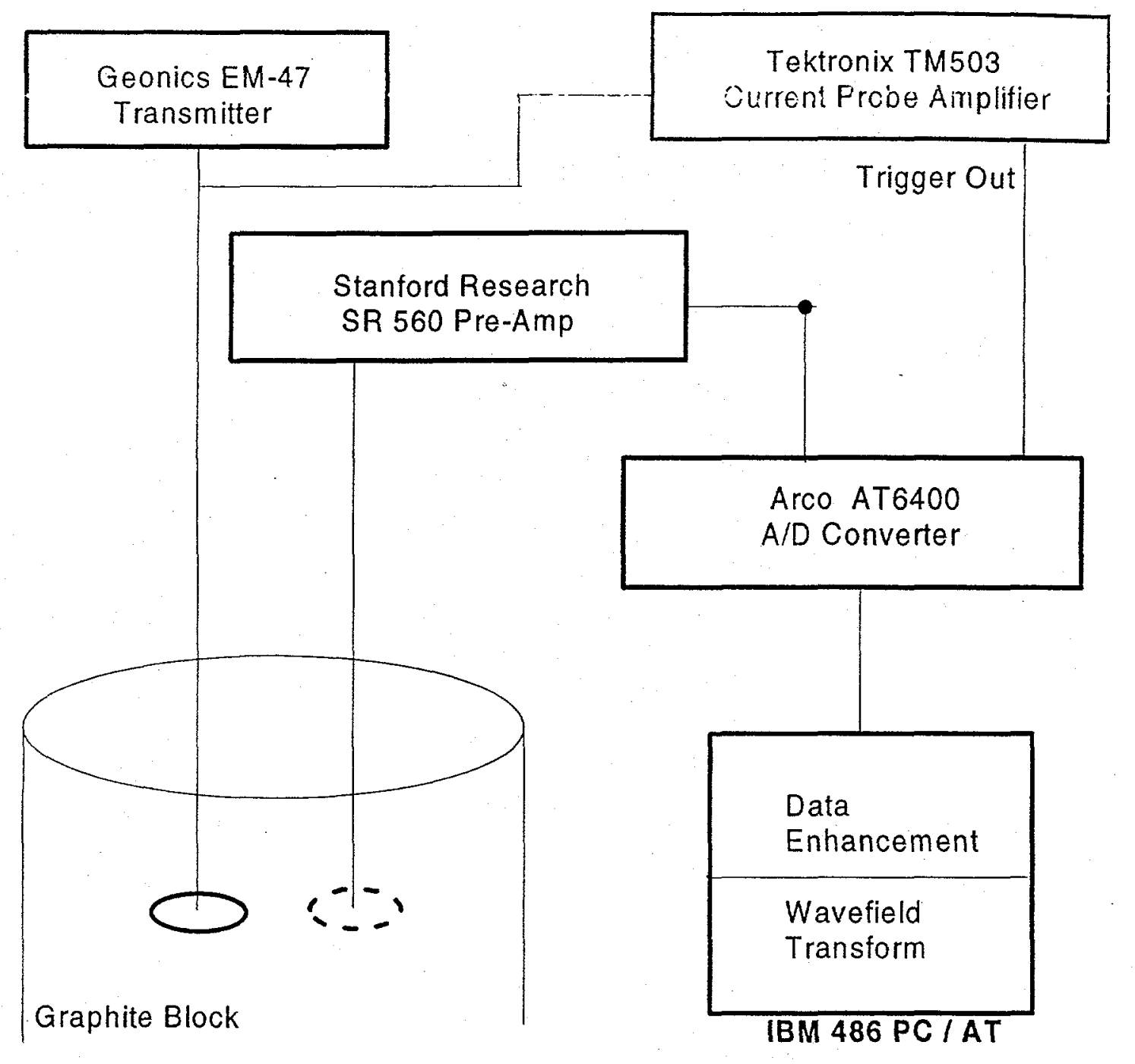

$\longrightarrow$ Transmitter

$\because-\because$ Receiver

Figure 3.1 Block diagram of the laboratory setup 


\subsection{Signal Processing}

The post acquisition data processing in this experiment can be broadly divided into two categories, viz. signal enhancement and wavefield transformation. The signal enhancement stage results in a significant improvement in the data quality and signal-tonoise ratio before the data is transformed to the wavefield domain. Two procedures are used in the first stage and the first of these is stacking. Generally 1000 traces are stacked and the average value obtained. This leads to the expected improvement in the signal-tonoise ratio by a factor of about 30 . The second one is time-windowing where the acquired data are averaged over a given time span which is possible because of the difference in the frequency content of the signal at early and late times. At early time the signal contains a lot of high frequency components At late time, however, when the signal is very weak, low frequency components are dominant and the signal follows a simple power law decay. This form of signal decay allows the use of variable width time windows over which the signal is averaged. These are held to an 8 microsecond width at the start and are progressively increased to a width of 160 microseconds. Since the background noise is of higher frequency than the signal, this process, which is equivalent to applying a low pass filter, gets rid of the noise without any loss of information content. The improvement in signal quality due to time-windowing is illustrated in figure 3.2. The details regarding the design of the windows are given in greater detail by Bentley (1993). Both the averaging and windowing procedures are discussed by Becker (1987). 

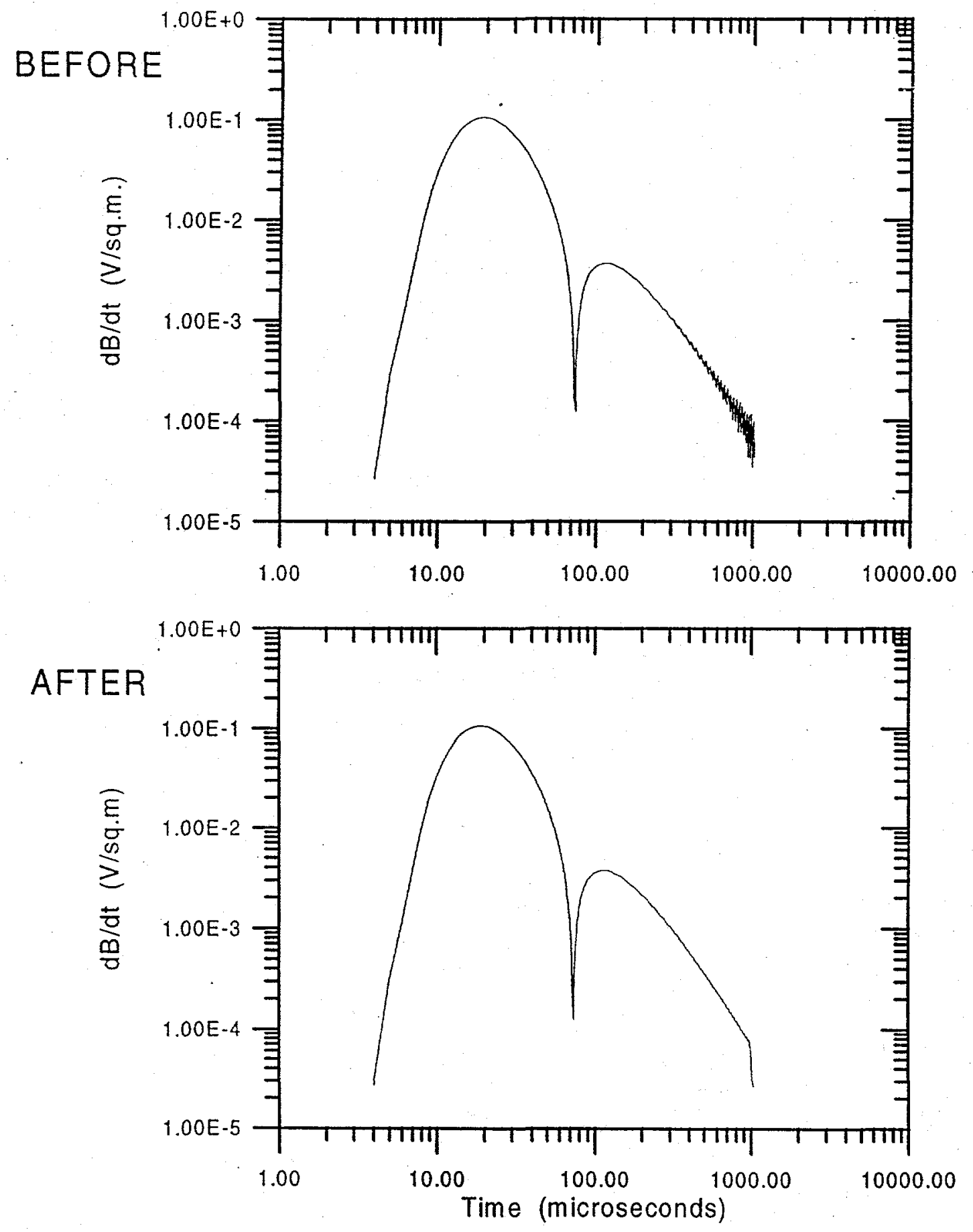

Figure 3.2 Effect of time-windowing on data 


\subsection{Signal Fidelity}

\subsubsection{Bandwidth}

Data quality requirements for the wavefield transformation are specified in terms of the signal duration and a maximum distortion value for the data in that time span. It is instructive to examine what these requirements translate to in the frequency domain. It turns out that the data requirements impose very stringent and specific bandwidth requirements on the system.

To a first approximation the receiver can be represented by the critically damped circuit shown in figure 3.3. Its impulse response is

$$
R(t)=\frac{w_{0}}{a}\left(w_{0} t e^{-w_{0} t}\right)
$$

where $w_{0}=$ resonant angular frequency of the receiver and

$\mathrm{a}=1+\mathrm{r} / \mathrm{R}$, a circuit parameter.

Although the input function or the primary magnetic field is a step function, the receiver sensor measures the rate of change in the magnetic field $(\mathrm{dB} / \mathrm{dt})$ and the output is the impulse response of the system. Following Ward and Hohmann (1987) the impulse response of a homogenous whole-space is given by

$$
\frac{\partial B}{\partial}=\frac{4}{\pi^{3 / 2}} \cdot \frac{m}{\sigma r^{5}} x^{-\frac{5}{2}} \cdot e^{-\frac{1}{x}}\left(1-\frac{1}{x}\right) \text { in } V / m^{2}
$$




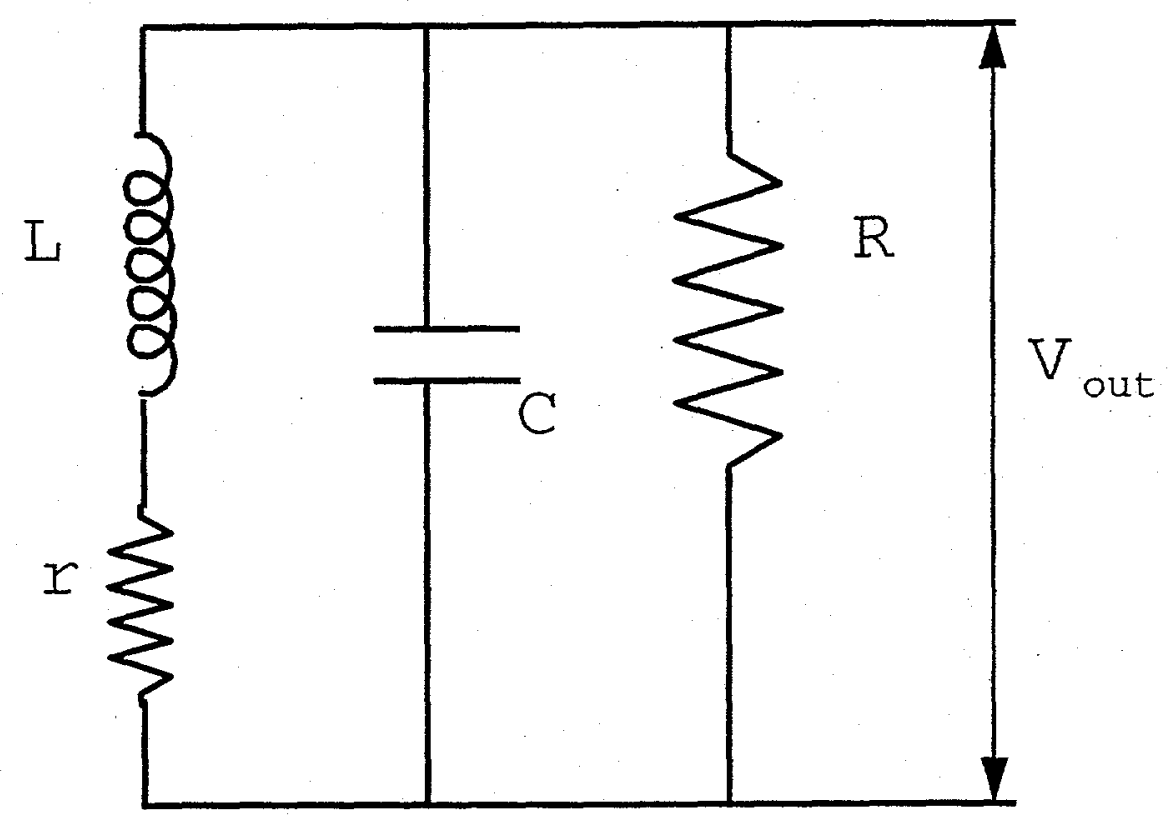

Figure 3.3 Receiver Circuit 
where

$\mathrm{m}=$ magnetic moment of source in Amp-sq. m;

$\sigma=$ medium conductivity in $\$ / \mathrm{m}$;

$r=$ receiver-transmitter separation in $\mathrm{m}$;

$\mathrm{x}=$ diffusion parameter defined by $\mathrm{x}=\mathrm{t} / \mathrm{T}$

where

$\mathrm{t}=$ time in seconds; and

$\mathrm{T}=\frac{\mu \sigma r^{2}}{4}$, the diffusion time in the medium.

In order to study the effect of the receiver bandwidth on the system response we must first scale the time factor by modifying the receiver impulse response to read $R(x)=\frac{w_{0}}{a} k x e^{-k x}$ with $k=w_{0} T$

The system response can then be represented as a convolution of the receiver response with the earth response (from equation 3.2 ) and is given by

$\mathrm{g}(\mathrm{x})=\frac{4}{\pi^{3 / 2}} \cdot \frac{m}{x^{5}} \cdot \frac{w_{0} k}{a} \cdot \int_{-\infty}^{\infty} u^{-5 / 2} e^{-1 / u}\left(1-\frac{1}{u}\right)(x-u) e^{-k(x-u)} d u$

Basically this is only a function of $\mathrm{k}$ and $\mathrm{x}$. The factor that determines signal fidelity is " $\mathrm{k}$ " i.e. the dimensionless product of the diffusion time in the host medium and the resonant angular frequency of the critically damped sensor.

To examine the matter let us consider a particular case. Let the coils be in the graphite scale model at an equal depth from the surface and at a lateral separation of five $\mathrm{cm}$. The 


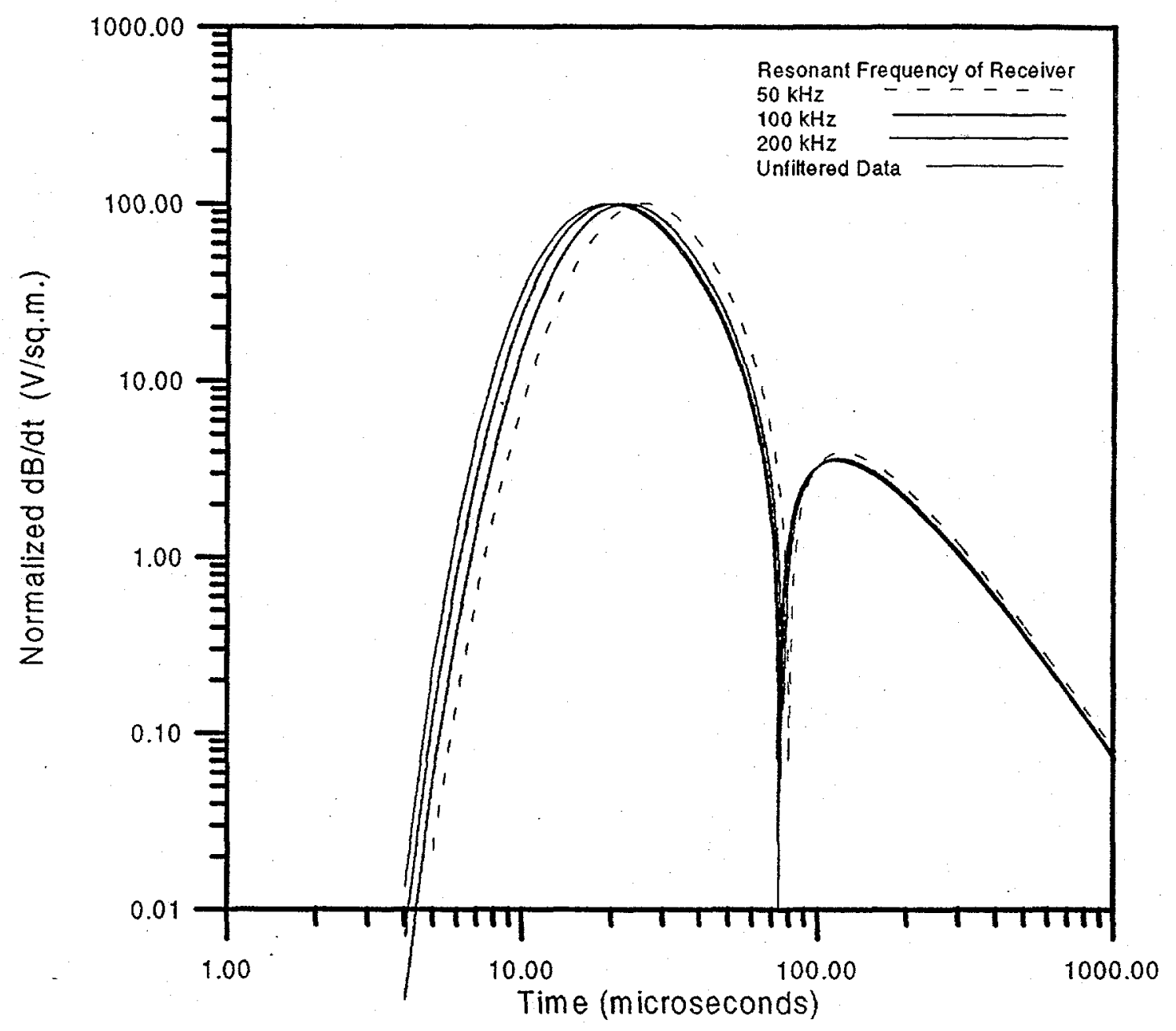

Figure 3.4 Effect of system bandwidth in time domain 
diffusion time in this instance is 74 microseconds. Figure 3.4 shows the undistorted signal in the time domain along with the output that would be obtained if the resonant frequency of the receiver was decreased from $200 \mathrm{kHz}$ through $50 \mathrm{kJz}$. In this illustration we see that, for the scale model, one can expect the output will closely represent the ground response if the receiver resonant frequency is $200 \mathrm{kHz}$ or greater. Conversely the ground response will be severely affected if that parameter is lowered to $50 \mathrm{kHz}$.

The results of limited bandwidth can be easily quantified in the wave domain i.e. after applying the wavefield transform to the data of figure 3.4 . In figure 3.5 it is shown that the undisturbed ground transient gives the expected arrival time in the wave domain. The . output using a receiver with the resonant frequency of $200 \mathrm{kHz}(\mathrm{k}=93)$ has the same arrival time as the undisturbed transient in the wave domain. But the output that would be recorded with a sensor with a lower resonant frequency gives a delayed arrival in the wave domain. The delay in $\mathrm{q}$ is $12 \%$ for the $100 \mathrm{kHz}$ resonant frequency sensor $(k=46.5)$ and $80 \%$ for the $50 \mathrm{kHz}(\mathrm{k}=23.2)$ resonant frequency sensor.

A better qualitative understanding of the bandwidth requirements can be obtained by examining this matter in the frequency domain. The pertinent data are shown in figure 3.6 which displays the receiver responses for sensors of different resonant frequencies as well as the ground response transformed to the frequency domain. Figure 3.6 (a) shows the amplitude responses while figure 3.6 (b) shows the phase of the receiver responses. Although the amplitude response, even for a $50 \mathrm{kHz}$ receiver, seems to encompass the signal spectrum we can see that the receiver with a resonant frequency of $50 \mathrm{kHz}$ will 


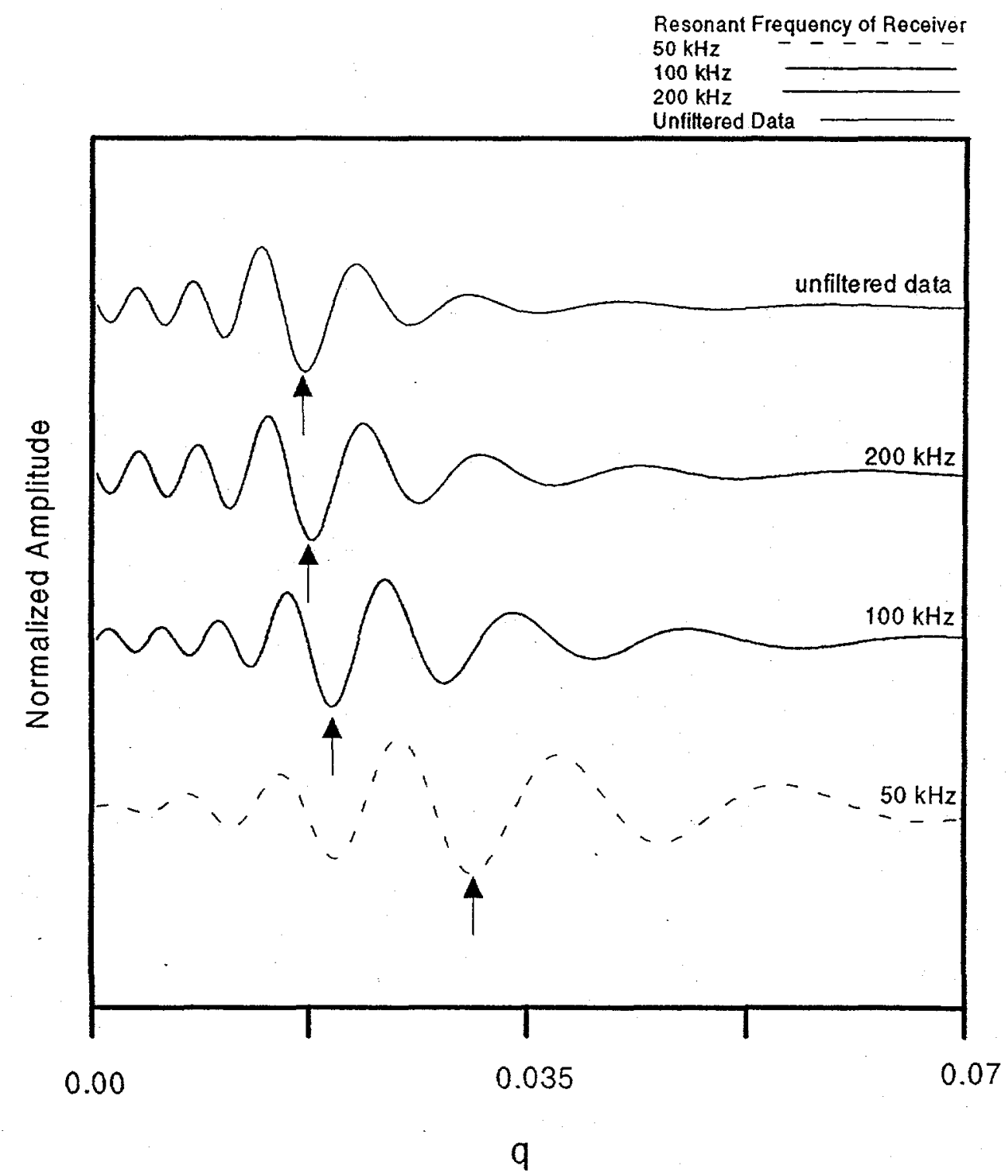

Figure 3.5 Effect of system bandwidth in wave domain 


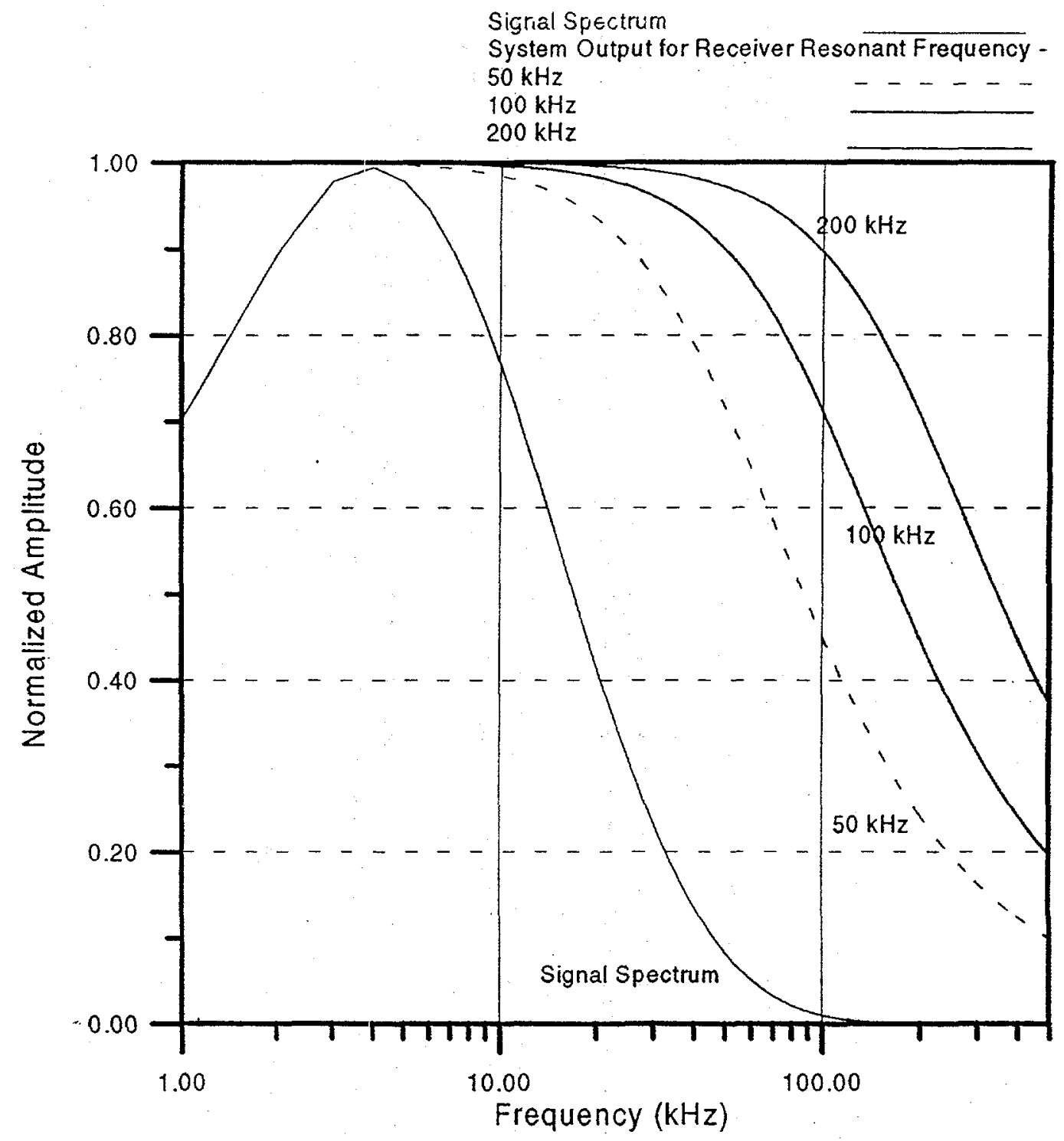

Figure 3.6 (a) Effect of system bandwidth in frequency domain - Amplitude 


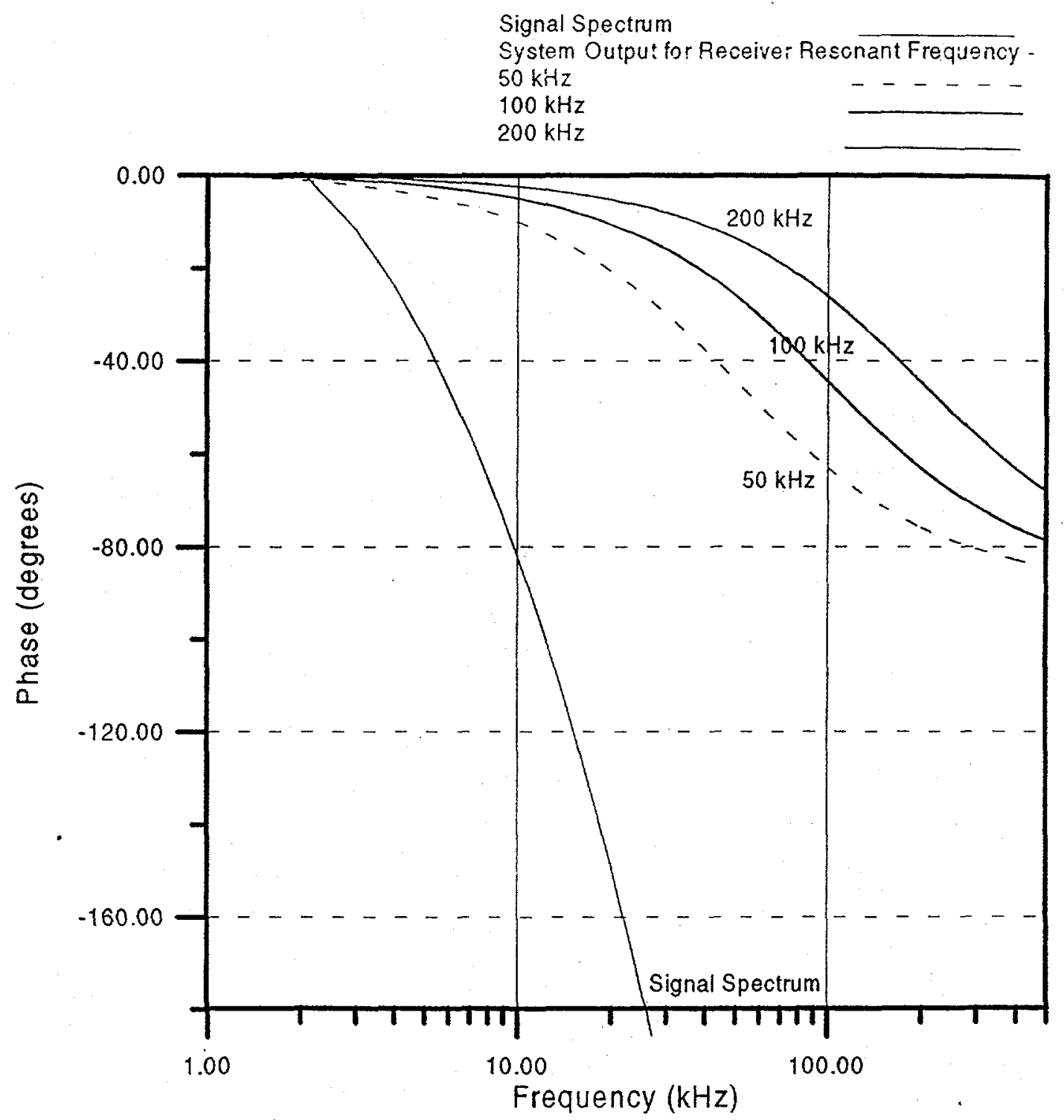

Figure 3.6 (b) Effect of system bandwidth in frequency domain - Phase 
distort the phase response of the observed signal severely when it is convolved with the ground response. A receiver with a $200 \mathrm{kHz}$ resonant frequency, however, will not introduce distortions in phase. Recalling that in this case the diffusion time in graphite was about $74 \mu s$ and that the minimal resonant frequency of the sensor required for distortionless response is about $200 \mathrm{kHz}$ we can conclude that " $\mathrm{k}$ ", the filter parameter, must be kept at a value greater than 100 .

In addition to the sensor characteristics any deviation in the transmitter waveform from a boxcar function also distorts the signal which no longer represents a step response. The effect of the ramp time on the signal is identical to that of a moving window average (Fitterman, 1987). That means high frequency components in the signal would be distorted. Thus the ramp time of the input signal has to be minimized and no harsh irregularities should be present.

\subsubsection{External Noise}

Apart from signal distortion the factor which affects fidelity is external noise. The requirements for the time domain signal as specified in Lee and Xie (1993) use a noise model in which the noise is a fixed percentage of the signal. The noise level encountered in this experiment, however, does not vary with the strength of the signal but stays constant over time except in very early times. This implies that the presence of noise imposes a minimum signal strength below which a reliable signal cannot be recorded. Therefore a more realistic evaluation of the noise present is given by the ratio of the 
maximum level of signal recorded to the minimum level. For this system such a figure which can be called the signal-to-noise ratio is $72 \mathrm{~dB}$.

External noise has been minimized by paying great attention to the cabling, grounding and shielding (Bentley, 1993). The transmitter and receiver cables are shielded using silver paint connected to a grounding wire. This method of shielding was experimentally found to be the most effective as it is not so thin as to offer a resistance which will produce a voltage large enough to be felt outside the barrier of the shield. At the same time it is not thick enough to support currents of sufficient magnitude to produce magnetic fields that will induce a voltage in the receiver. The electrostatic coupling cannot be completely eliminated even with the shielding and the resulting noise renders the part of the signal before five microseconds useless.

\subsubsection{Data Quality}

The tests carried out to check data quality include repeatability and reciprocity checks and comparisons with theoretical predictions. The data was found to be repeatable to within 1\%. In any electromagnetic measurement, results should remain unchanged if the transmitter and receiver are interchanged. The result of such a crosswell reciprocity check is shown in figure 3.7. The transmitter and receiver are in different boreholes in the graphite block. In these experiments the transmitter-receiver configuration has been interchanged while all other parameters have been left unchanged. The zero-crossing of the signal in the time domain was found to be very sensitive to any signal distortion. In this case the two datasets are equivalent and cross zero at the same time. While the 


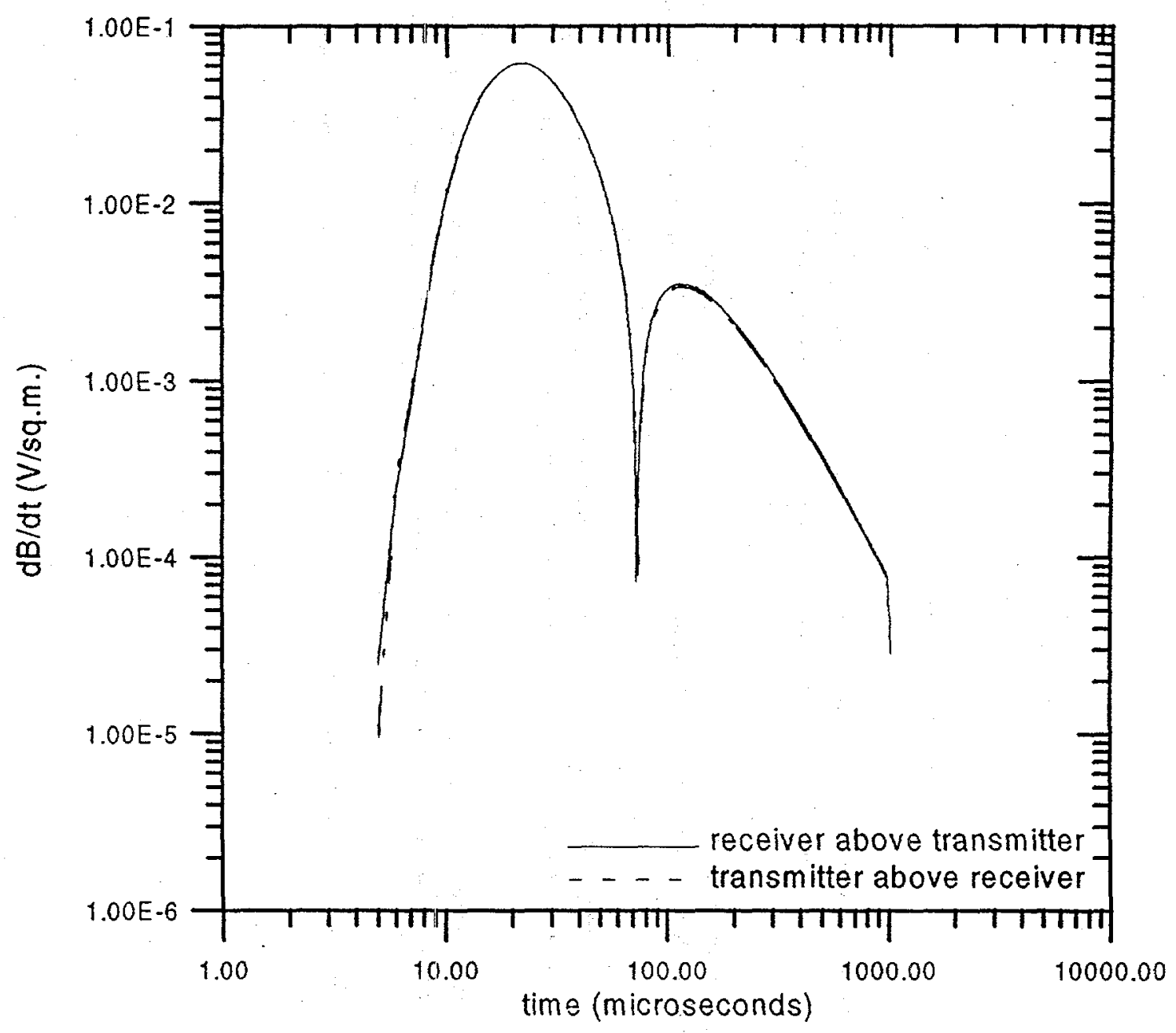

Figure 3.7 Reciprocity test in cross-borehole configuration vertical separation between sensors $=2 \mathrm{~cm}$ 
amplitudes of the signals vary they overlap when one is laid over the other with an rms error of less than $0.6 \%$. This shows that the coil separation is being recorded accurately

Figure 3.8 shows a comparison of the theoretical and experimental data with the amplitudes scaled for plotting purposes. The transmitter and receiver are at the same vertical level in this case at an interwell distance of $5 \mathrm{~cm}$. There is an excellent match between the theoretical and experimental data. Again two signals cross zero at the same time. For times later than five microseconds the difference between the two signals at any point is less than $1 \%$.

\subsubsection{Sensor Orientation}

In the course of the reciprocity tests we observed that sensor rotation about its axis had an observable effect on the time domain data. After some deliberations this was ascribed to the fact that the sensor axis was not exactly parallel to the borehole axis. Precise sensor orientation is crucial for accurate measurement of the magnetic field. Figure 3.9 (a) shows the effect a 90 degree rotation of the receiver coil about its axis has on the data. In this case the transmitter and receiver are placed in graphite at a vertical separation of $4 \mathrm{~cm}$ and a horizontal separation of $5 \mathrm{~cm}$. The observed time domain waveforms are clearly different. In the " $q$ " domain, however, the traveltime results are invariant. Figure 3.9 (b) shows the two datasets transformed to the wave domain where we see that the arrival is at the same value of $q$. Therefore the conductivity of the medium can be accurately extracted from the wave domain data even when coil rotations change the data in time. 


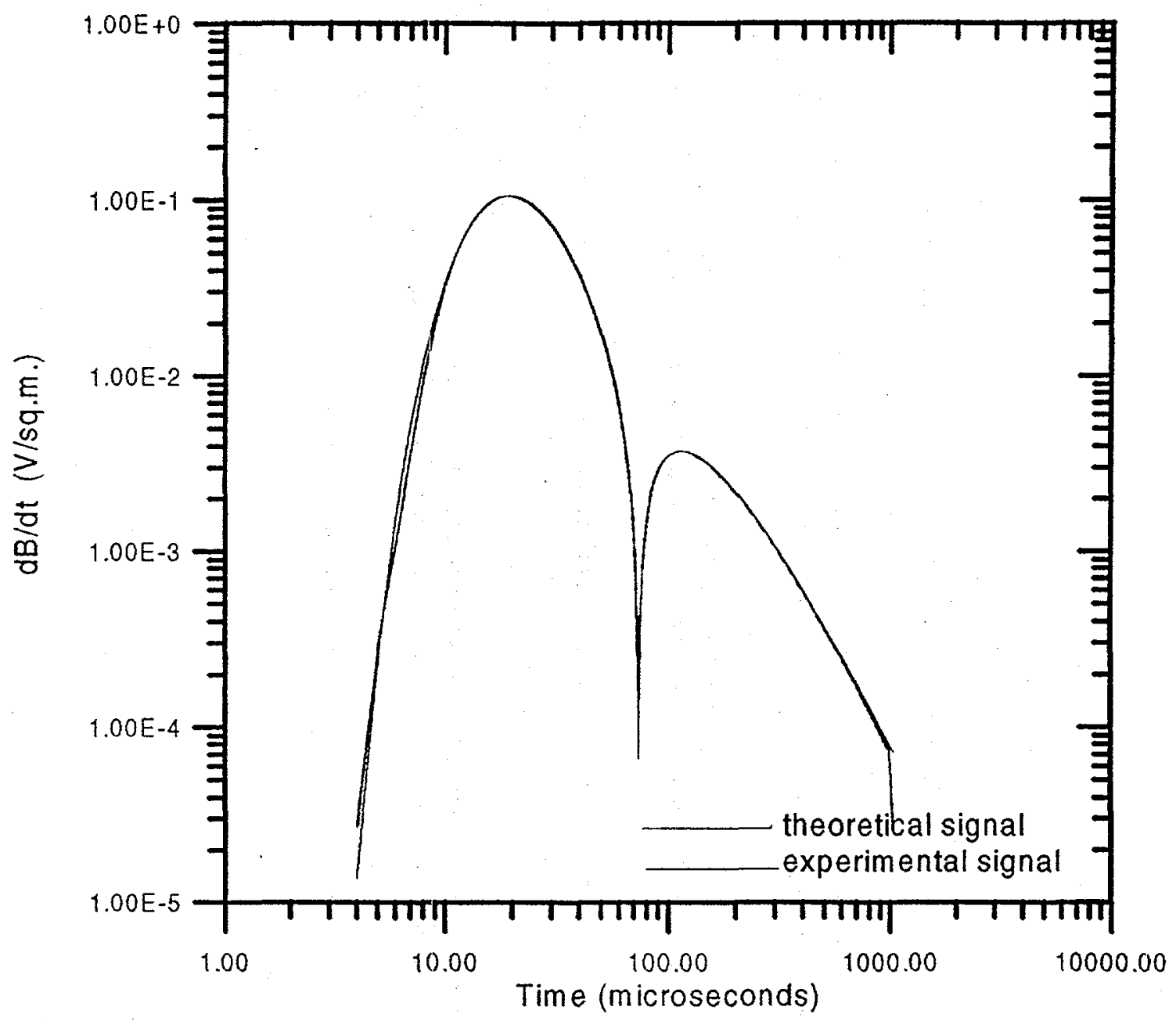

Figure 3.8 Comparison between theory and experiment 


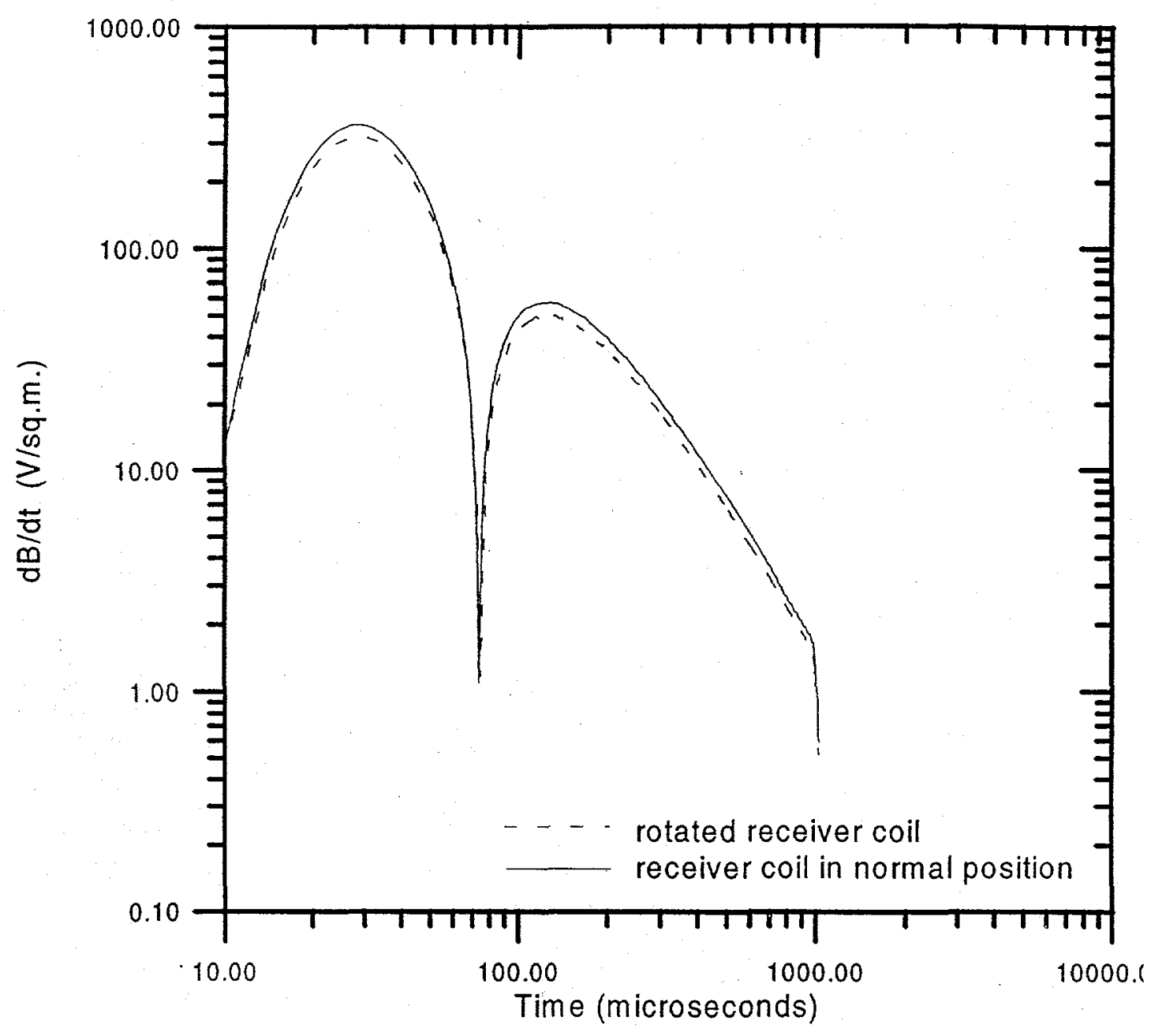

Transmitter depth $-10 \mathrm{~cm}$

Receiver depth $-14 \mathrm{~cm}$

Fig.3.9 (a) Effect of rotation of receiver coil in time domain 


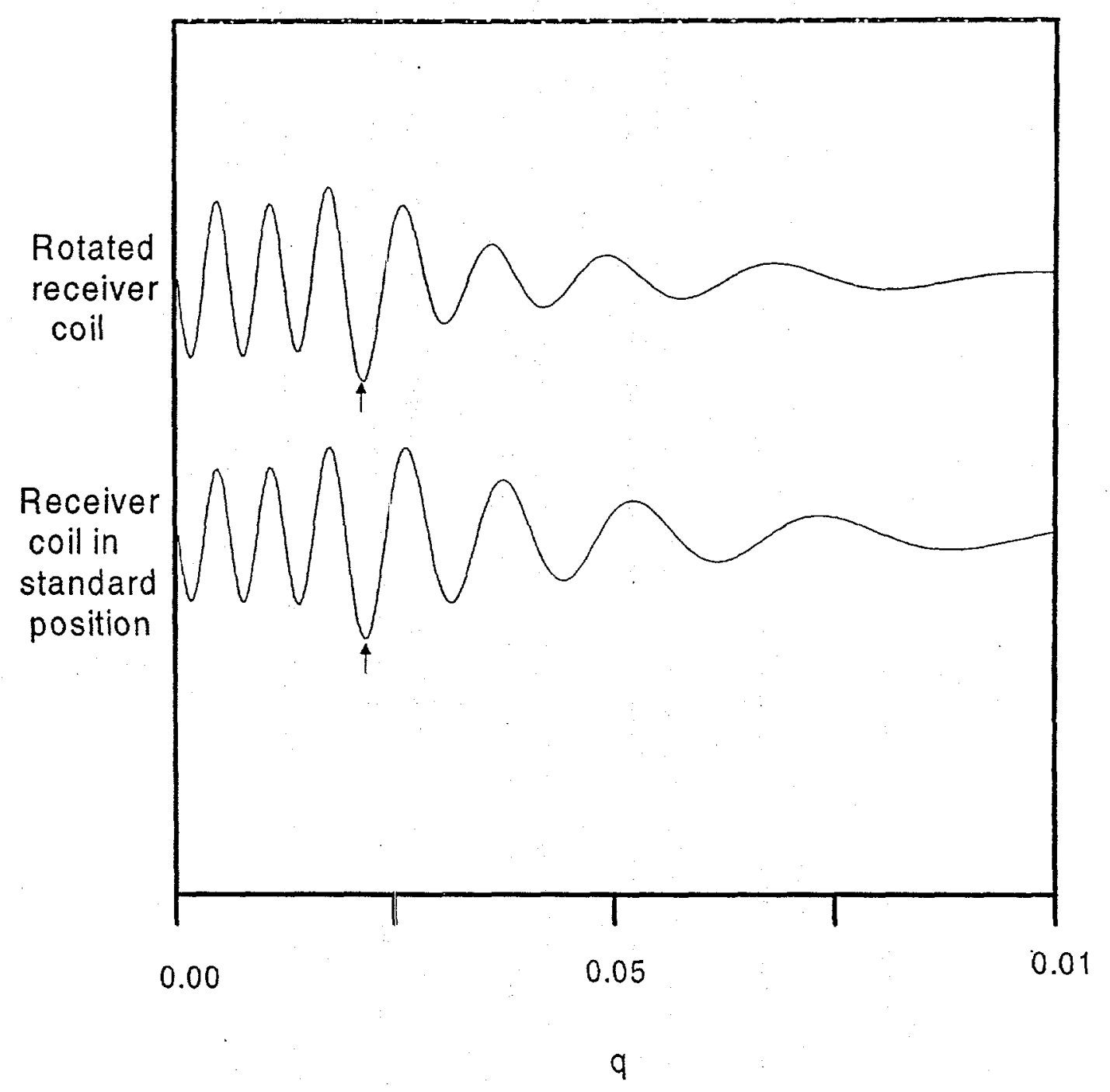

Transmitter depth $-10 \mathrm{~cm}$

Receiver depth $-14 \mathrm{~cm}$

Figure 3.9 (b) Effect of rotation of receiver coil in wave domain 
This provides for great robustness in the use of the wavefield transform technique for conductivity estimation.

\section{RESULTS}

Four experiments were done using the scale model. Two of these were carried out on the graphite blocks which were used to simulate a homogenous earth. For the other experiments one or more steel sheet layers were inserted between the two graphite blocks so as to create a three layer earth model. In the first three experiments the transmitterreceiver configuration is analogous to the seismic common shot point gather where the source and receivers are located in adjacent vertical boreholes. The last experiment was done using a borehole-to surface configuration.

The data are obtained in the time domain and transformed to the wave domain. In the wave domain the traveltime in " $q$ " is determined by the arrival of the maximum amplitude point of the wave. The theoretical expression for the arrival in $q$ is given by 
$q_{0}=\frac{r}{v}=r \sqrt{\mu \sigma}$

where $v=$ wave velocity;

$\mathrm{r}=$ distance traveled by wave $(\mathrm{m})$;

$\mu=$ magnetic permeability;

$\sigma=$ electrical conductivity

The travelpath of the wave is calculated using simple ray theory. Within one medium the wave is assumed to travel directly from the source to the receiver in a straight line. At the interface between two layers the ray is taken to obey Snell's law. In the following discussion the experimentally obtained arrival times are compared to the theoretical ones. As discussed in section 2.1 the resolution in the wave domain is limited by the size of the $\mathrm{q}$-step in the transformation program. The increment in $\mathrm{q}$ is $3.6 \times 10^{-4} \sqrt{\sec \text { onds }}$ for these experiments.

4.1 Cross-borehole experiment in uniform medium

Let us first examine the simulation of a cross-borehole sounding. This experiment is done in graphite blocks. The system configuration is shown in figure 4.1 (a). The transmitter is kept at the depth of $10 \mathrm{~cm}$ below the surface while the receiver position is varied from a depth of 6 to $14 \mathrm{~cm}$ with a reading being taken at every $2 \mathrm{~cm}$. The horizontal distance between the boreholes is $5 \mathrm{~cm}$. 
Transmitter in fixed position

Receiver position varies

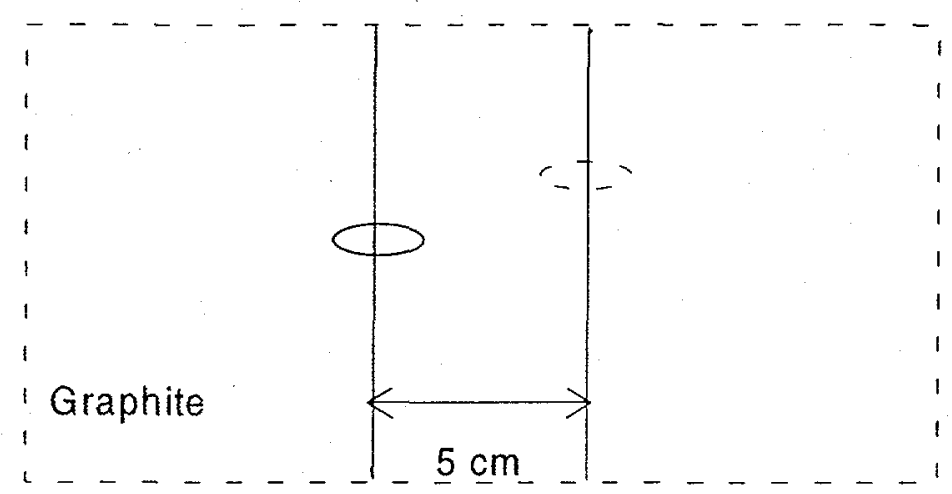

$\longrightarrow$ Transmitter

... Receiver

Figure 4.1 (a) Cross-borehole dataset - system configuration

VERTICAL SECTION SHOWN - NOT TO SCALE 


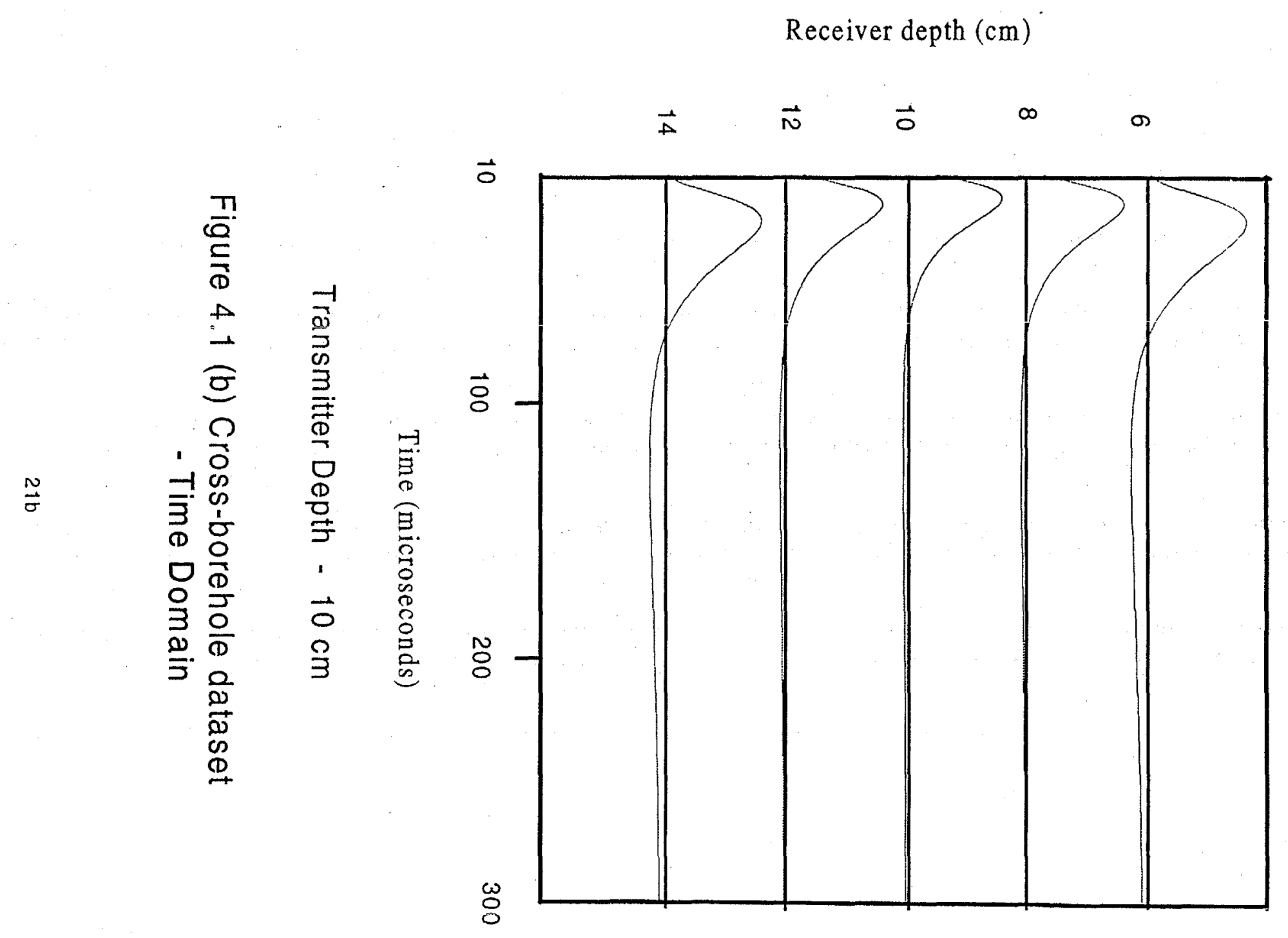


The time domain results are shown in figure 4.1 (b). When the receiver is at a depth of 10 $\mathrm{cm}$ it is nearest to the transmitter. As the receiver moves away in either direction the signal takes longer to diffuse and crosses zero later. When transformed to the " $q$ " domain the observations can be assembled in the form of a conventional "common source gather" crosswell seismogram (figure 4.1 (c)). It becomes very clear from this figure that the transmitter is closest to the receiver when it is $10 \mathrm{~cm}$ below the surface. In all the cases except when the receiver is farthest from the source the agreement with theory is within one q-step. In other words the accuracy of the data is limited only by the resolution limits imposed by the transformation program. Thus, the error is less than $2 \%$. In the case when the receiver is nearest to the surface the error is two qsteps or $3.2 \%$. The actual arrivals are shown in the table below.

\section{TABLE 4.1 DIRECT WAVEFIELD ARRIVALS FOR EXPERIMENT 4.1}

\begin{tabular}{|l|l|l|l|l|l|l|l|}
\hline $\begin{array}{l}\text { Transmitter } \\
\text { Depth }\end{array}$ & $\begin{array}{l}\text { Receiver } \\
\text { Depth }\end{array}$ & \multicolumn{2}{l|}{ Observed Arrival } & \multicolumn{2}{l|}{ Predicted Arrival } & \multicolumn{2}{l|}{ Error } \\
\hline & & $\sqrt{s}$ & q-steps & $\sqrt{s}$ & q-steps & $\%$ & q-steps \\
\hline 10 & 6 & 0.0227 & 63 & 0.0020 & 61 & 3.2 & 2 \\
\hline 10 & 8 & 0.0188 & 52 & 0.0185 & 51 & 1.4 & 1 \\
\hline 10 & 10 & 0.0172 & 48 & 0.0172 & 48 & 0.7 & 0 \\
\hline 10 & 12 & 0.0188 & 52 & 0.0185 & 51 & 1.4 & 1 \\
\hline 10 & 14 & 0.0224 & 62 & 0.0020 & 61 & 1.8 & 1 \\
\hline
\end{tabular}




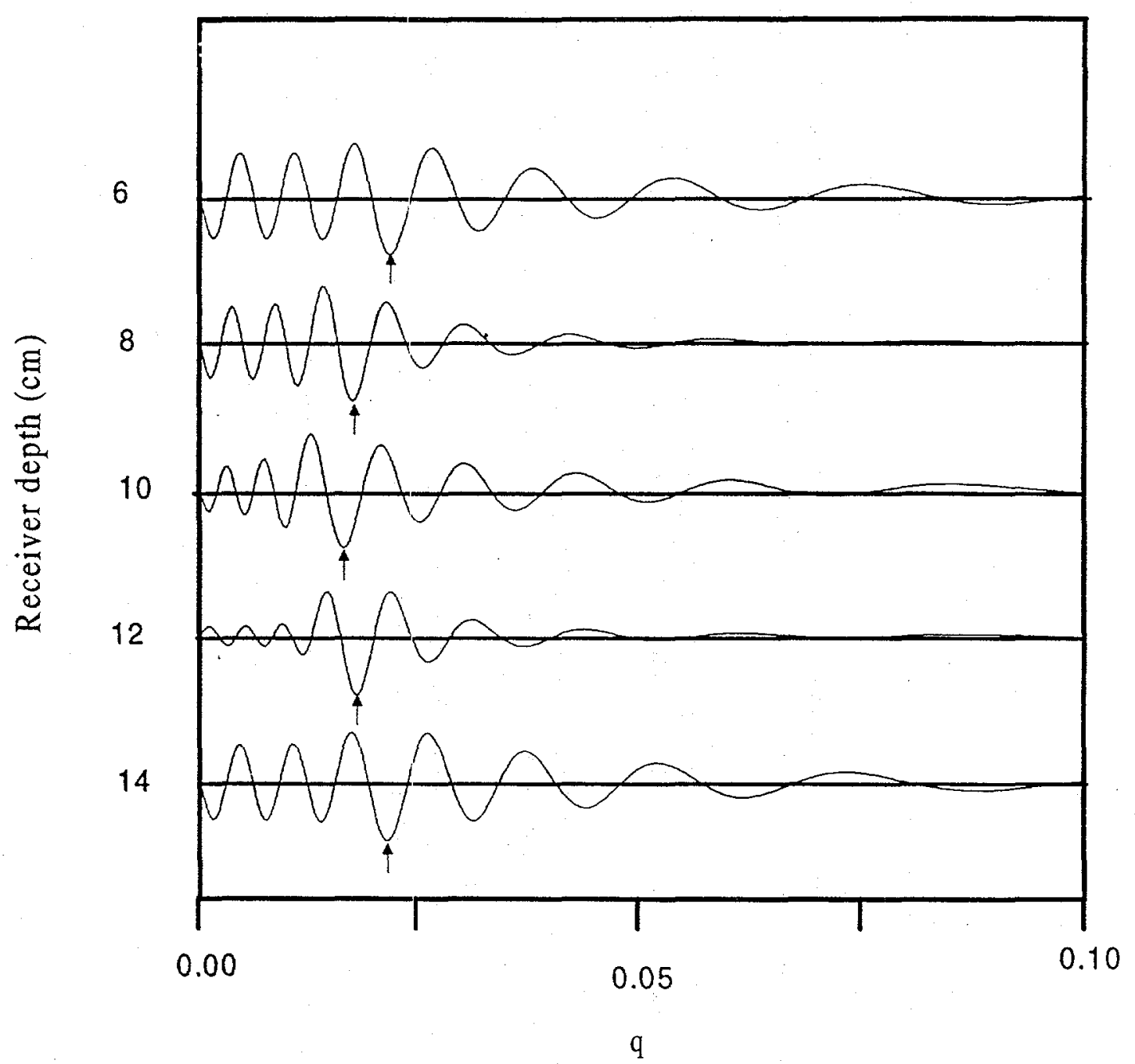

Transmitter Depth - $10 \mathrm{~cm}$

Figure 4.1 (c) Cross-borehole dataset - Wave Domain 
4.2 Cross-borehole experiment across highly conducting thin sheet

In this experiment a steel sheet with a conductance of 1668 Siemens was put between two graphite blocks to simulate a three layer earth. The system configuration is shown in figure 4.2 (a). The transmitter is kept fixed at a depth of $16.5 \mathrm{~cm}$ from the surface or 1.5 $\mathrm{cm}$ below the sheet while the receiver is in the other borehole above the sheet. Readings are taken at three positions of the receiver at a spacing of $1 \mathrm{~cm}$.

The time domain results are shown in figure 4.2 (b). The results with the same system configuration but without the steel sheet are shown in dotted lines. The introduction of the steel sheet slows down the diffusion of the signal as a result of eddy currents set up in the sheet. But the effect of the steel sheet is much more distinct and easily quantifiable in the wavefield transformed data (figure 4.2 (c)). It is obvious that the direct arrival of the wavefront is delayed by the conductive sheet where the speed of the wave is slower than the surrounding medium. In all the three positions of the receiver the match with the theoretical values is within the resolution limit of one q-step. Therefore the error is less than $3 \%$. 
Transmitter position is fixed Receiver position varies

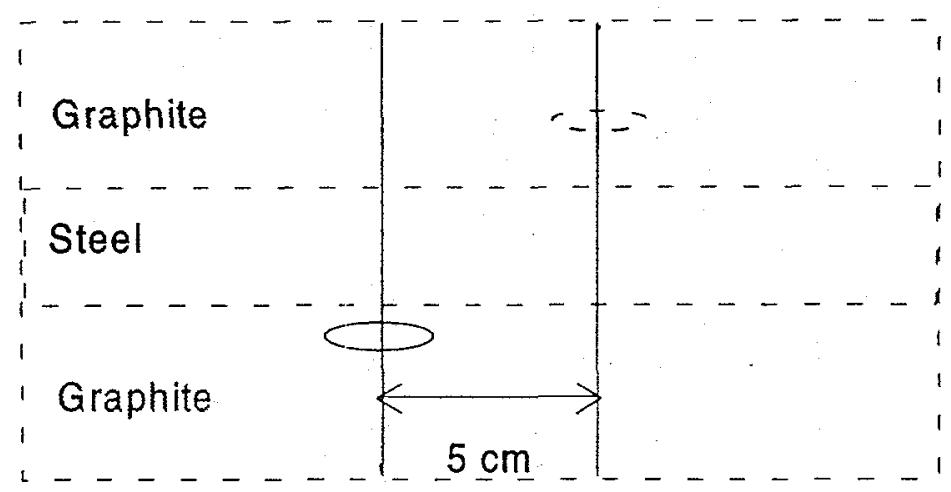

$\longrightarrow$ Transmitter

_._. Receiver

- Steel layer is $0.12 \mathrm{~cm}$ thick.

Figure 4.2 (a) Cross-borehole dataset across highly conducting thin sheet - system configuration

VERTICAL SECTION SHOWN - NOT TO SCALE 


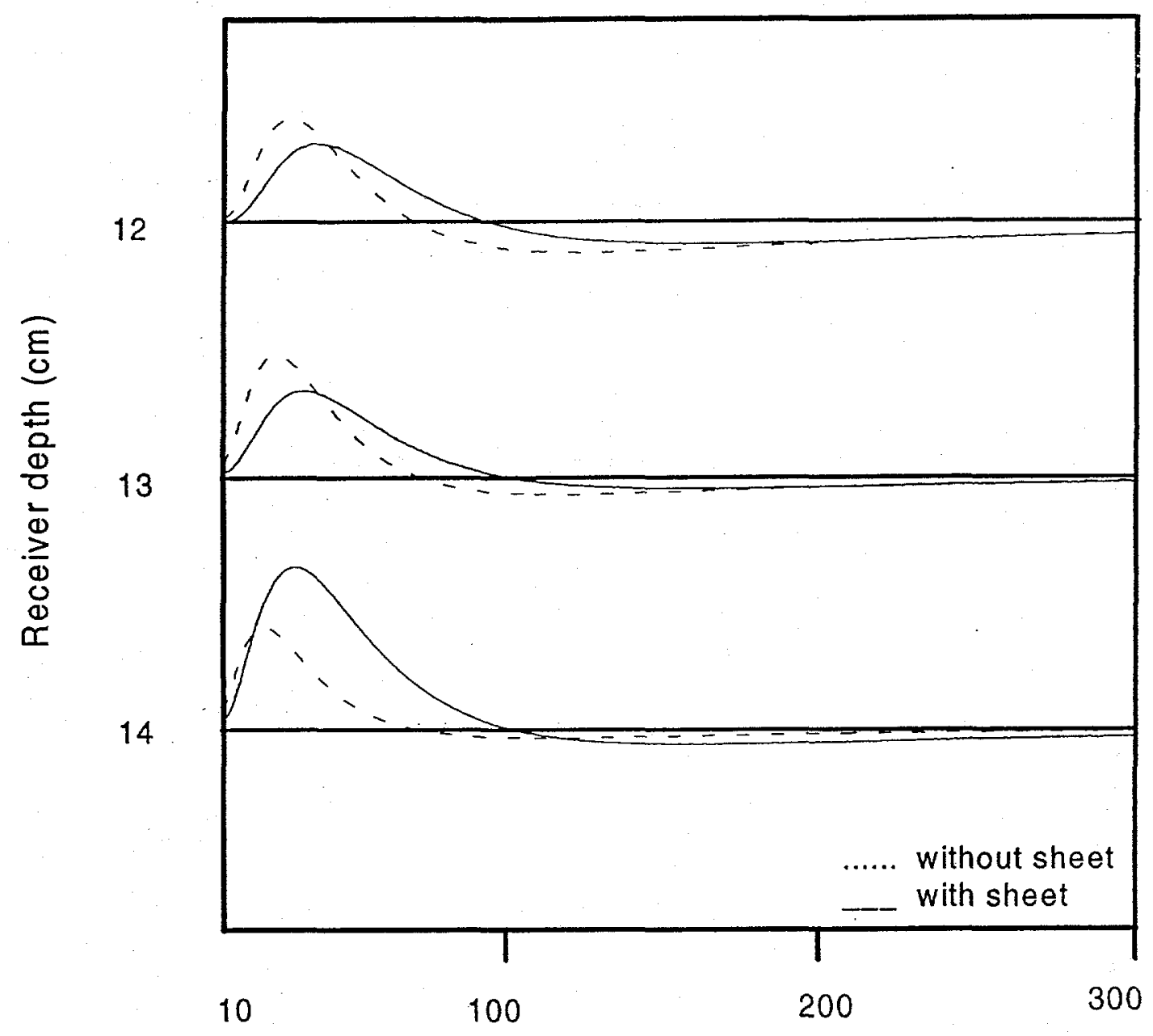

Time (microseconds)

Interwell distance - $5 \mathrm{~cm}$

Transmitter depth $-16.5 \mathrm{~cm}$

Figure 4.2 (b) Cross-borehole data across highly conducting thin sheet - Time Domain 


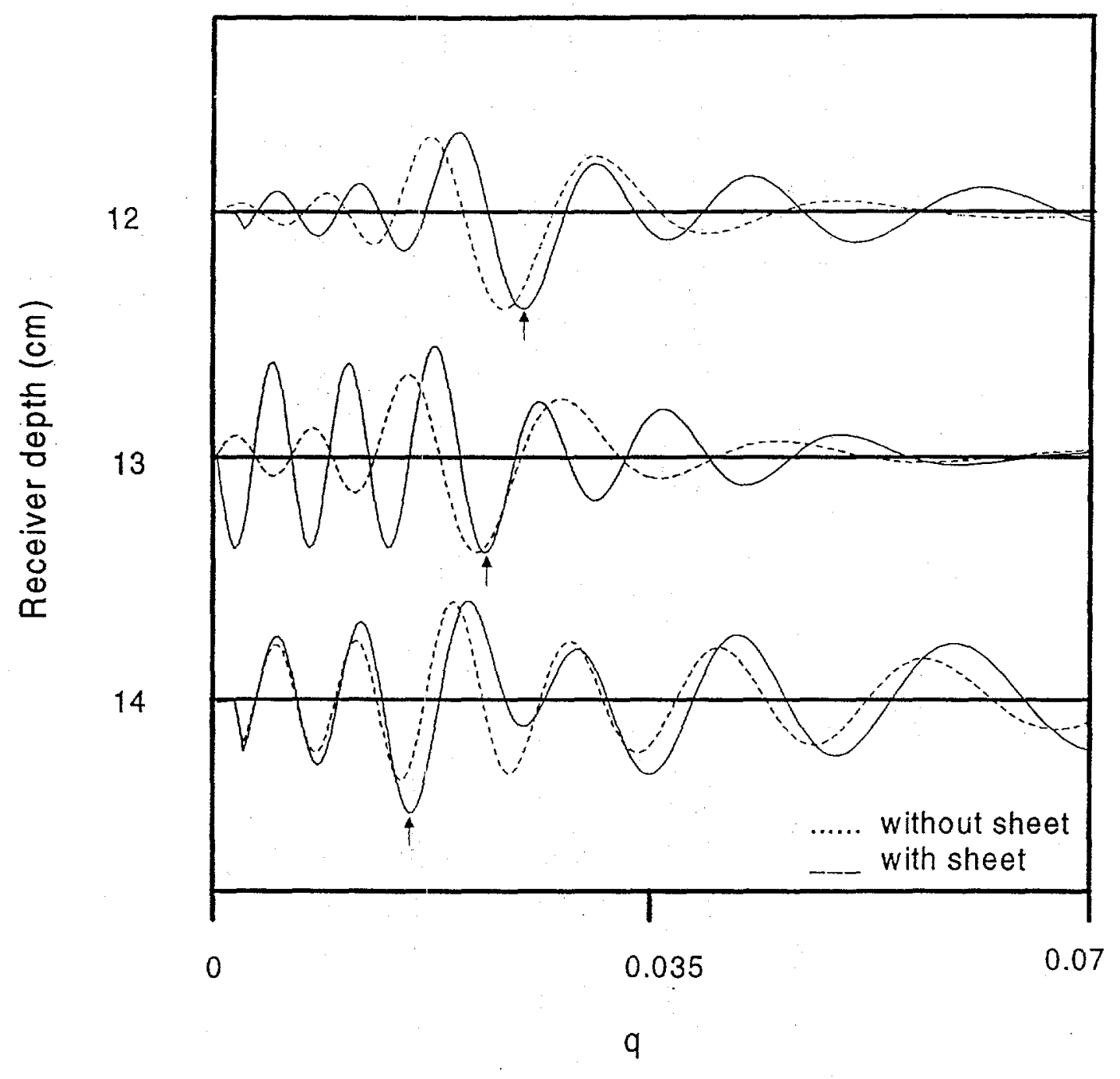

Interwell distance - $5 \mathrm{~cm}$

Transmitter depth $-16.5 \mathrm{~cm}$

Figure 4.2 (c) Cross-borehole data across highly conducting thin sheet - Wave Domain 
TABLE 4.2 DIRECT WAVEFIELD ARRIVALS FOR EXPERIMENT 4.2

\begin{tabular}{|l|l|l|l|l|l|l|l|}
\hline $\begin{array}{c}\text { Transmitter } \\
\text { Depth }\end{array}$ & $\begin{array}{l}\text { Receiver } \\
\text { Depin }\end{array}$ & \multicolumn{2}{|l|}{ Observed Arrival } & \multicolumn{2}{l|}{ Predicted Arrival } & \multicolumn{2}{l|}{ Error } \\
\hline & & $\sqrt{s}$ & q-steps & $\sqrt{s}$ & & $\%$ & q-steps \\
\hline 16.5 & 12 & 0.02503 & 69 & 0.02471 & 68 & 1.3 & 1 \\
\hline 16.5 & 13 & 0.02322 & 64 & 0.22570 & 63 & 2.9 & 1 \\
\hline 16.5 & 14 & 0.02177 & 60 & 0.02186 & 61 & -0.4 & -1 \\
\hline
\end{tabular}

4.3 Cross-borehole experiment across a variable thickness conducting sheet

In this experiment a three-layer earth is simulated again but the thickness of the middle layer is varied with the help of a stack of $1700 \mathrm{~S}$ steel sheets. The system configuration is shown in figure 4.3 (a). The positions of the transmitter and receiver are kept constant in this experiment. The transmitter is at the same position as in the previous experiment, viz, $1 \mathrm{~cm}$ below the lowermost steel layer. The receiver is $14 \mathrm{~cm}$ below the surface which is 1 . $\mathrm{cm}$ above the uppermost steel layer. So the transmitter-receiver separation is $2.5 \mathrm{~cm}$ in addition to the thickness of the steel layer.

The time domain results are shown in figure 4.3 (b). The first signal is through graphite before putting any steel sheet between the two blocks. This signal passes through a graphite-air interface but the effect of that is negligible. In other words the graphite-air interface is undetectable. Then the experiment is repeated with one, two and three steel 
Transmitter $1 \mathrm{~cm}$ above steel layer Receiver $1.5 \mathrm{~cm}$ below steel layer Thickness of steel layer varies

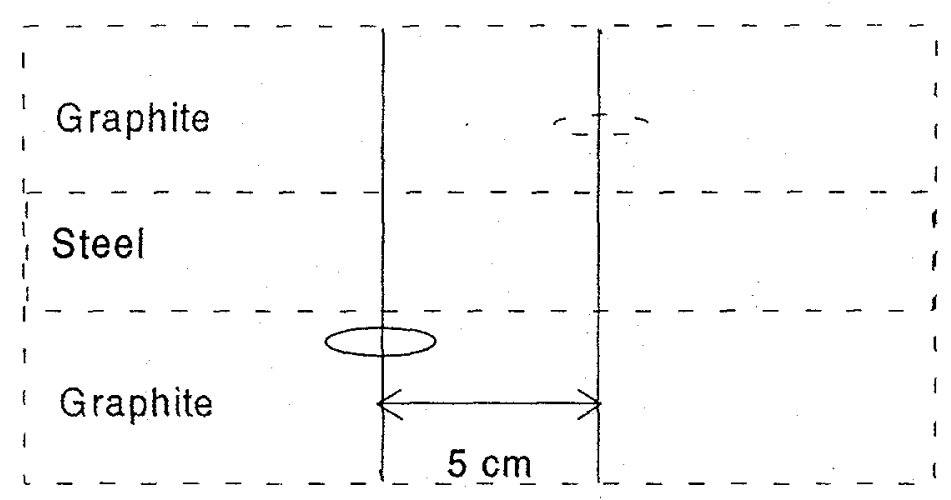

$\longrightarrow$ Transmitter

_._. Receiver

Figure 4.3 (a) Cross-borehole dataset across variable thickness steel sheet - system configuration

VERTICAL SECTION SHOWN - NOT TO SCALE 


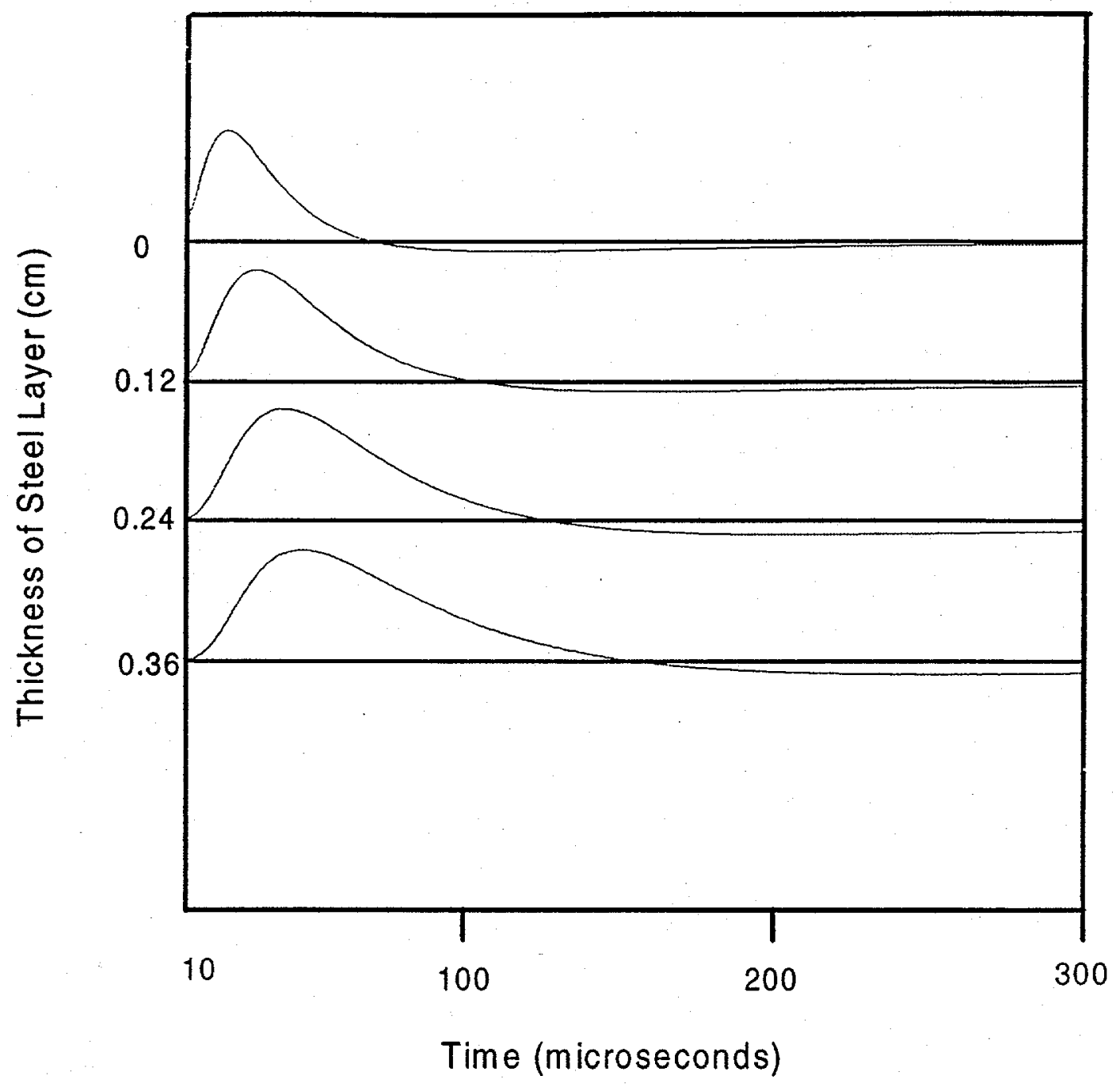

Figure 4.3 (b) Cross-borehole dataset across variable thickness steel sheet - Time Domain 
sheets respectively constituting the middle layer between the two graphite blocks. All the sheets are of the same thickness. The zero-crossing of the signal is delayed as the thickness of the steel layer is increased. The arrivals in the wave domain demonstrate the linear increase in the thickness of the layer much more clearly as can be seen in figure $4.3^{\circ}$ (c). The arrival of the wave gets delayed when the first conductive sheet is introduced. This delay doubles when two sheets are used and is tripled when three sheets are used. The difference between the experimental and theoretically calculated data in all these cases is less than one q-step. The error in each case is $2.1 \%$ or less.

TABLE 4.3 DIRECT WAVEFIELD ARRIVALS FOR EXPERIMENT 4.3

\begin{tabular}{|l|l|l|l|l|l|l|}
\hline $\begin{array}{l}\text { Thickness } \\
\text { of middle } \\
\text { layer(cm) }\end{array}$ & \multicolumn{2}{l|l}{ Observed Arrival } & \multicolumn{2}{l|}{ Predicted Arrival } & \multicolumn{2}{l|}{ Error } \\
\hline & $\sqrt{s}$ & q-steps & $\sqrt{s}$ & q-steps & $\%$ & q-steps \\
\hline 0 & 0.02068 & 57 & 0.02028 & 57 & 2 & -1 \\
\hline 0.12 & 0.02177 & 60 & 0.02186 & 60 & -0.4 & -1 \\
\hline 0.24 & 0.02394 & 66 & 0.02345 & 66 & 2.1 & 1 \\
\hline 0.36 & 0.0254 & 70 & 0.02504 & 70 & 1.4 & 1 \\
\hline
\end{tabular}




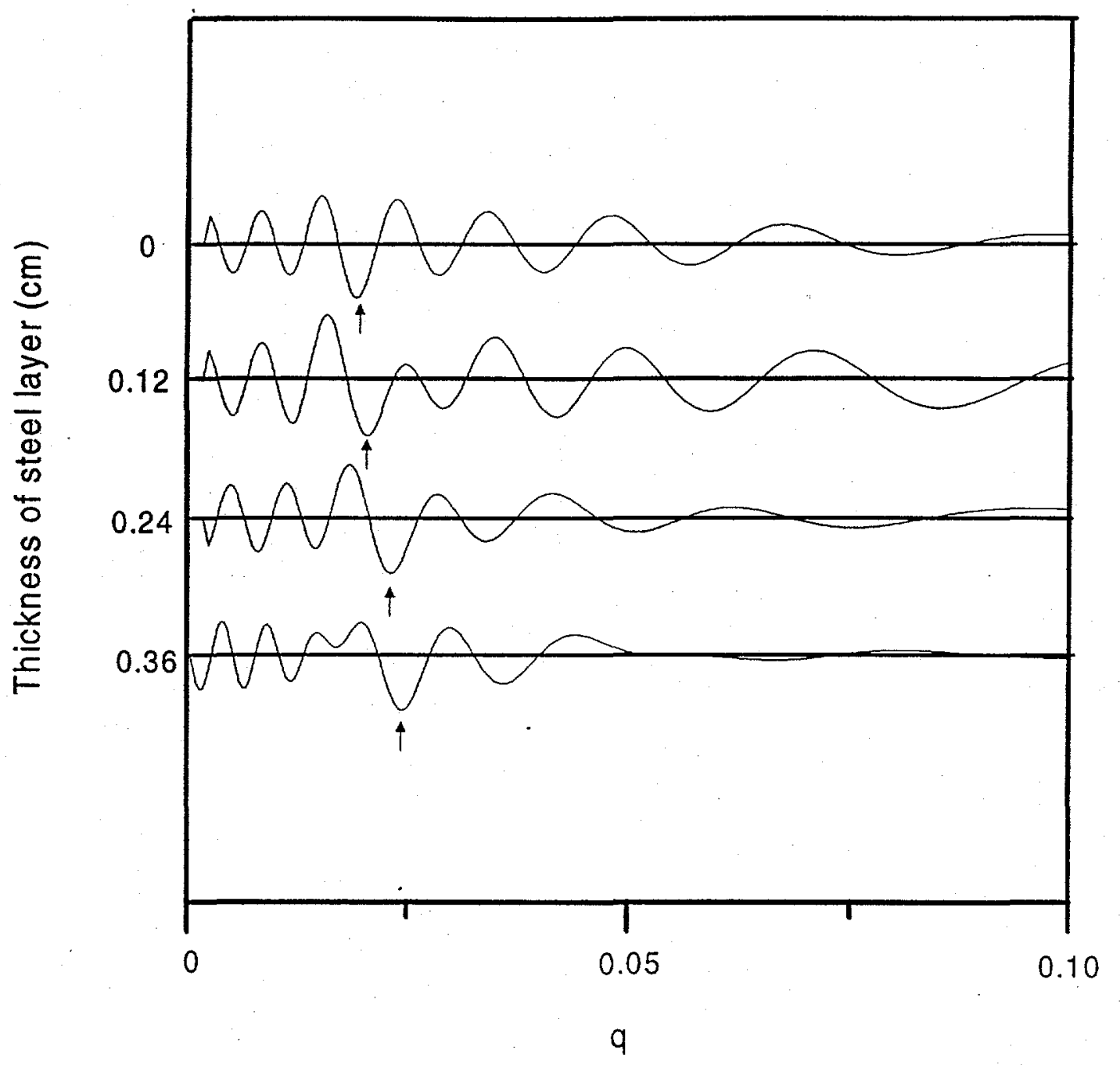

Figure 4.3 (c) Cross-borehole dataset across variable thickness steel sheet - Wave Domain 
4.4 The surface-to-borehole experiment

The most exciting experimental results are shown in figure 4.4 . These were obtained in the surface-to-borehole configuration for which the theoretical treatment has not yet been formulated. The receiver is kept at the surface (shown in figure 4.4(a)) and the transmitter is kept in a borehole at a horizontal distance of $5 \mathrm{~cm}$ from the receiver. When the transmitter is at the surface the signal that reaches the receiver travels through the air. This can be seen in the time domain data (figure 4.4 (b)) which shows the observed signals as the transmitter is moved down into the borehole. It can be seen that the signal peaks later and crosses zero later as the coil separation increases. In the wave domain the ray path formulation still holds. Figure 4.4 (c) shows the corresponding wave domain signals. The first signal arrival, with both the transmitter and the receiver at the surface, does not correspond to its theoretical value. But all the other signals do and the increasing delay of the direct arrival as the trarımitter descends corresponds to the increasing travel path of the wave. As shown in table 4.4 , in this experiment the correspondence between the theoretical and experimental values is less exact than previously. The maximum error is of 3 q-steps or $3.8 \%$ when the transmitter-receiver separation is the greatest. But the validity of the wave domain formulation beyond the theoretically predicted boundaries is of great importance. 
Receiver in fixed position

Transmitter position varies

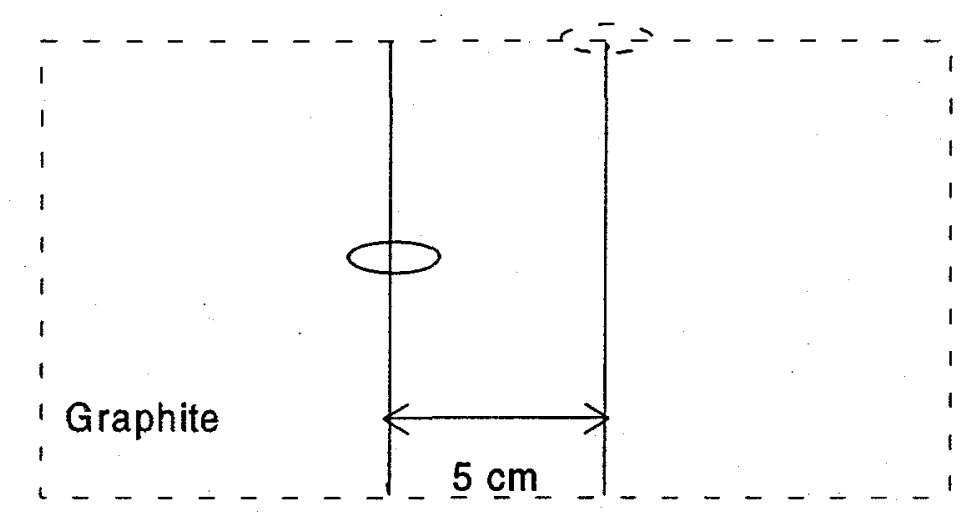

$\varnothing$ Transmitter

(-.- Receiver

Figure 4.4 (a) Borehole-to-surface dataset

- system configuration

VERTICAL SECTION SHOWN - NOT TO SCALE 


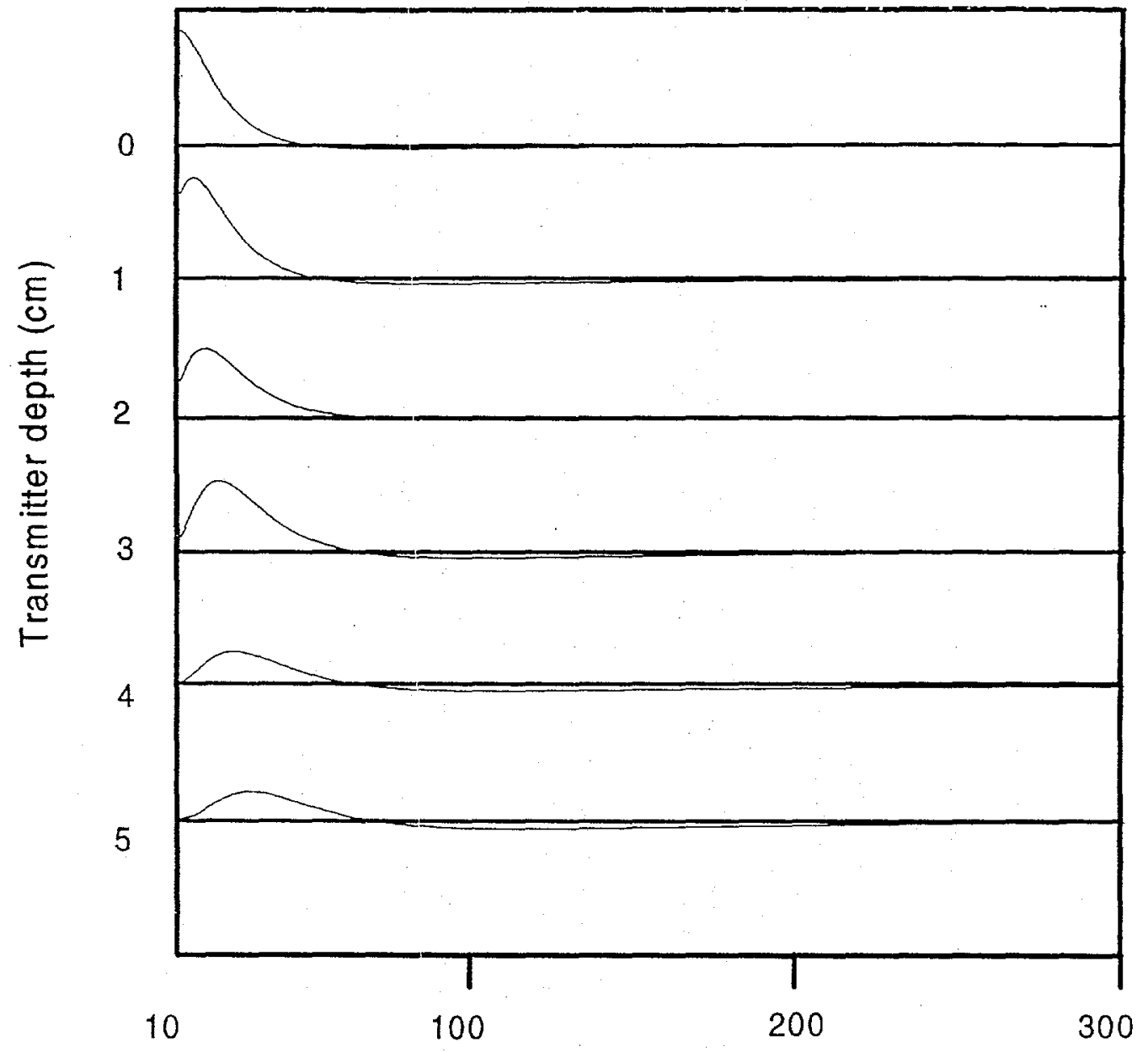

Time (microseconds)

Receiver at surface at a distance of $5 \mathrm{~cm}$ from survey well

Figure 4.4 (b) Borehole-to-surface data

- Time Domain 


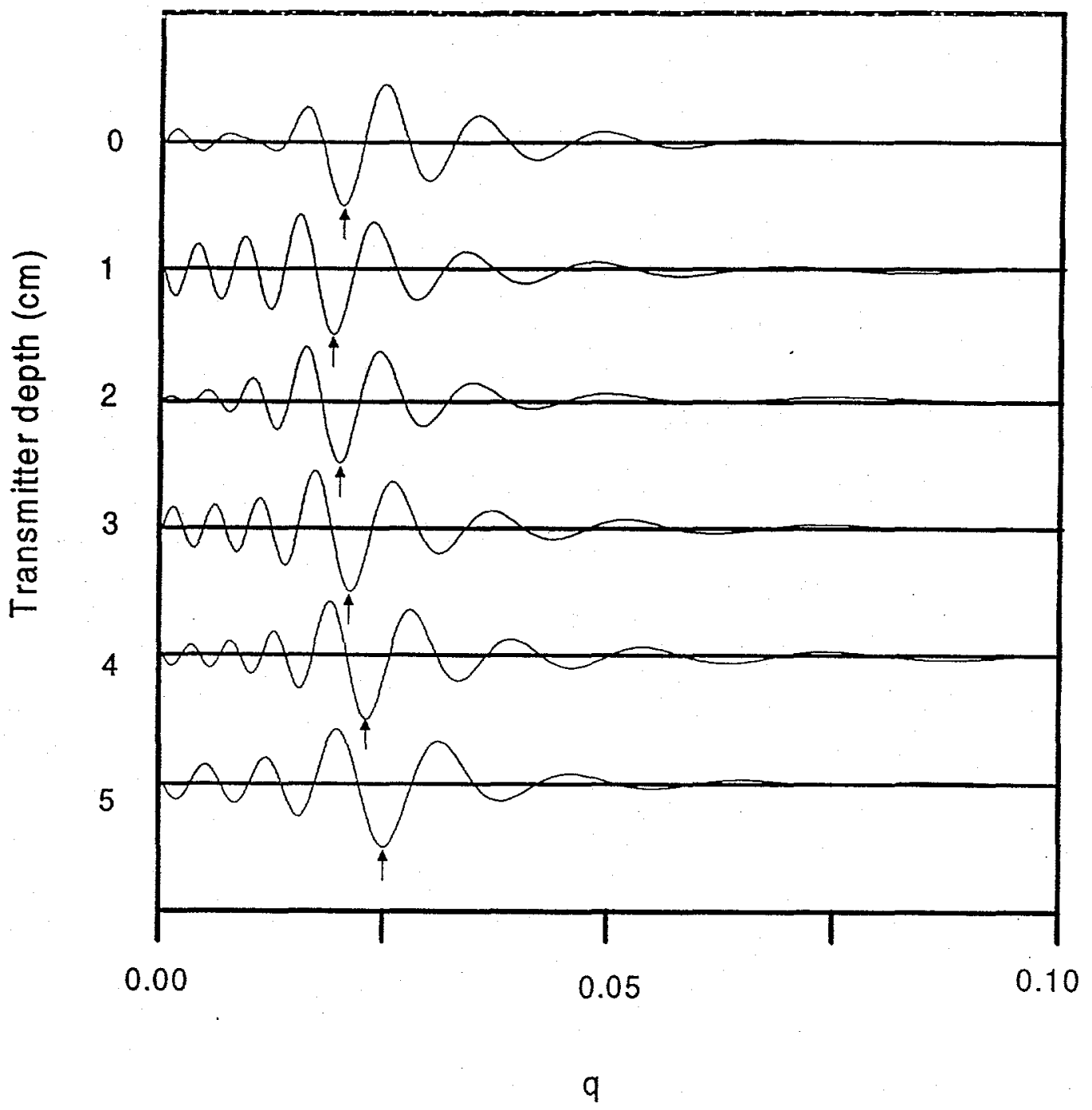

Receiver at surface at a distance of $5 \mathrm{~cm}$ from survey well

Figure 4.4 (c) Borehole-to-surface data - Wave Domain 
TABLE 4.4 DIRECT WAVEFIELD ARRIVALS FOR EXPERIMENT 4.4

\begin{tabular}{|l|l|l|l|l|l|l|l|}
\hline $\begin{array}{l}\text { Transmitter } \\
\text { Depth }\end{array}$ & $\begin{array}{l}\text { Receiver } \\
\text { Depth }\end{array}$ & \multicolumn{2}{|l|}{ Observed Arrival } & \multicolumn{2}{l|}{ Predicted Arrival } & \multicolumn{2}{l|}{ Error } \\
\hline & & $\sqrt{s}$ & q-steps & $\sqrt{s}$ & q-steps & $\%$ & q-steps \\
\hline 2 & 0 & 0.01696 & 47 & 0.01752 & 48 & 3.3 & -1 \\
\hline 3 & 0 & 0.01840 & 51 & 0.01851 & 51 & 0.6 & 0 \\
\hline 4 & 0 & 0.01949 & 54 & 0.02004 & 56 & 2.8 & -2 \\
\hline 5 & 0 & 0.02165 & 60 & 0.02200 & 61 & 1.6 & -1 \\
\hline
\end{tabular}

\section{CONCLUSIONS}

The experiments carried out using the graphite based scale model system establish the validity of the wavefield transform. This experiment has shown that time domain electromagnetic data acquired in a laboratory environment with a limited dynamic range of $72 \mathrm{~dB}$ and a corresponding limited time span of two decades (10-1000 $\mu$ s) can be successfully transformed to the wavefield domain. 
This project has also made possible the laying down of minimum requirements for data acquisition systems required for making observations that are amenable to wavefield analysis procedures. It is seen that system bandwidth is a crucial parameter. The sensor requirements can be best stated by the specification of a minimum " $k$ " of 100 where $k$ is the dimensionless product of the diffusion time in the host medium and the resonant angular frequency of the critically damped sensor. In addition to this the transmitter waveform has to be free of harsh irregularities and must have a short ramp time. Considerable insight into the characteristics of the wavefield transform has been gained. It was found that the transform is unaffected by small errors in sensor orientation which makes this technique very robust.

A realistic field situation was scaled down to laboratory dimensions and simulated using graphite blocks and steel sheets. Three cross-borehole simulations were conducted. The first experiment was in a homogenous graphite medium where the transmitter was kept in a fixed position in one borehole while the receiver was moved from a position above the transmitter to one below in another borehole. The results when transformed to the wave domain were within $2 \%$ of theoretical predictions except for the signals obtained at the maximum coil separation. In that case the error is $3.2 \%$. The second experiment was across a steel sheet used to simulate a highly conductive second layer in a three-layer earth model. The transmitter was kept at a fixed position below the sheet while the receiver was kept above the sheet in another borehole and moved downwards in $1 \mathrm{~cm}$ increments. The maximum error in this experiment is less than $3 \%$. In the third experiment the transmitter 
and receiver were kept in a fixed position in adjacent boreholes while the thickness of the steel sheet were varied. In this case the maximum error in traveltime in the wave domain is $2.1 \%$. The fourth experiment was a surface-to-borehole experiment which gave surprisingly accurate wave domain results considering that no theoretical formulation for such a situation exists. The maximum error in this case is $4.4 \%$.

Further work needs to be: targeted towards making the transform more robust in the presence of noise while improving resolution in the wavefield domain. Three different approaches towards that end seem feasible. The first is preprocessing the data so that the impulse response in the wavefield domain is obtained. Presently the impulse response in time domain is directly transformed to the wavefield domain. Either the source function in the time domain can be changed or the impulse response in the time domain can be numerically convolved with another function like a Gaussian pulse to get higher resolution in the wave domain. Numerical studies need to be carried out to select the optimal function for the source.

The second approach is to extend the range of the signal in time. The signal asymptotes to a power law at late times. Since the experimental data obtained so far reaches this asymptotic region it could possibly be extrapolated to extend its range. In cases where closed form analytical approximations for late time are not available other extrapolation techniques can be used. The use of adaptive filters for this purpose needs to be explored. Successful extrapolation of acquired data at both extremes of the time range would lead to possibilities for better resolution using singular value decomposition with a smaller 
regularization parameter. Accurate estimation of traveltime in the wave domain is a prerequisite to better resolution in conductivity imaging.

The third approach to improving resolution is further processing of the wave domain signal. The individual trace should be examined in its Fourier domain and appropriate filters should be designed to improve the signal-to-noise ratio by attenuating the noise. Since the noise in the data is amplified during the process of the wavefield transformation such an exercise could prove to be rewarding. We could also consider additional signal processing in the spatial domain. Thus, when a series of wave traces are obtained, say by varying the receiver position, we have a two-dimensional dataset and two dimensional signal processing techniques which are used in seismic data processing could then be used. Of particular interest are wavefield separation techniques which could result in the observation of reflections. Taking advantage of the fact that a wavefront which is transmitted or reflected from a layer boundary has a specific velocity which shows up as a straight line in $\mathrm{f}-\mathrm{k}$ space, powerful wavefield separation techniques can be used to extract transmitted and reflected wavefronts from noisy data. Here $f$ is the temporal frequency while $\mathrm{k}$ is the spatial frequency. Transformation to $\mathrm{f}-\mathrm{k}$ space is achieved by using a twodimensional Fourier transform on a dataset. Extensive trials have to be carried out to find the optimal techniques that would lead to maximum relaxation of data quality requirements while improving resolution. 
In conclusion it can be stated that the practical feasibility of the wavefield transformation technique has been established and the path has been cleared for progress to the field trial stage. 
References

Becker, A., Lee, K.H., Wang, Z., and Xie, G., 1994, Acquisition of precise electromagnetic data; EAEG $56^{\text {th }}$ Annual Meeting and Exhibition $/ 6^{\text {th }}$ EAPG Conference, Vienna, Austria, June 6-10.

Becker, A., and Cheng, G., 1987, Detection of repetitive electromagnetic signals; Ed. Misac N. Nabighian; Electromagnetic Theory in Applied Geophysics - Theory, 2 vols., Society of Exploration Geophysicists, Vol. 1.

Fitterman, D.V., and Anderson, W. L., 1987, Effect of transmitter turn-off time on transient soundings, Geoexploration, Vol. 24.

Gershenson, M., 1993, Simple interpretation of time-domain electromagnetic sounding using similarities between wave and diffusion propagation; Proceedings of 63rd SEG Annual International Meeting, Extended Abstract no.SS2.34, p.1342-1345.

Grant, F. S., and West, G.F., 1965, Interpretation Theory in Applied Geophysics, McGraw Hill.

Jia, P., Flockhart, I.W., and Wilson, A. J. S., 1995, Automatic parallelisation of multichannel transient EM processing; International Symposium on ThreeDimensional Electromagnetics, Schlumberger-Doll research, Ridgefield, CT, USA, Oct. 4-6.

Lee, K.H., 1989, A new approach to interpreting electromagnetic-sounding data; Annual Report 1988, Lawrence Berkeley Laboratory, LBL-26362; p.24-27.

Lee, K.H., Liu, G. and Morrison, H.F., 1989, A new approach to modeling the electromagnetic response of conductive media; Geophysics, Vol. 54, No.9 (September 1989), p1180-1992.

Lee, K.H. and Xie, G., 1993, A new approach to imaging with low-frequency electromagnetic fields; Geophysics, Vol. 58, p.780-796.

Slob, E.C., Habashy, T.M., and Torres-Verdin, C., 1995, A new stable numerical procedure for computing the q-transform of TEM data, EAGE $57^{\text {th }}$ Conference and Technical Exhibition, Proceedings, Glasgow, Scotland, May 29-June 2, 1995.

Ward, S. H., and Hohmann, G.W., 1987, Electromagnetic Theory for Geophysical Applications: Ed. Misac N. Nabighian; Electromagnetic Theory in Applied Geophysics - Theory, 2 vols., Society of Exploration Geophysicists, Vol. 1.

Wilson, A. J. S., Ziolkowsky, A.; Hobbs, B.A., and Sharrock, D.S., 1995, Time-lapse EM: 3D EM in practice; International Symposium on Three-

Dimensional Electromagnetics, Schlumberger-Doll research, Ridgefield, CT, USA, Oct. 4-6. 


\section{APPENDIX: THE USER MANUAL FOR THE WAVEFIELD TRANSFORM PROGRAM}

\section{Introduction}

The wavefield technique of analysis of electromagnetic data requires the application of the wavefield or q-transform on that data (Lee and Xie, 1993). This transforms the data from time-space into wavefield-space where the variable analogous to time is ' $q$ '. In this space or domain the first arrival of the waveform is taken to be the q value where the signal is of maximum positive amplitude. This value is given by

$q=r \times \sqrt{\mu \sigma}$

where $r=$ transmitter-receiver separation,

$\mu=$ magnetic permeability of the medium,

and $\sigma=$ electrical conductivity of the medium.

One quick and easy way of using the wavefield analysis is to obtain the conductivity of the medium as the transmitter-receiver separation distance is generally known.

\section{Input Files}

The flow chart of the transform code is included. The files fort.11 and emtq. dat have to be prepared before using the program. The electromagnetic data in the time domain is put in an ASCII file named fort.11. The format is two columns with time in the first column and the signal strength in the second column. This data is the impulse response of the system (or the $\mathrm{dB} / \mathrm{dt}$ recorded by the sensor when the transmitter input is a voltage step function). The input data has to be sampled at $1 \mathrm{MHz}$ and 1024 data points are required. The control file is named emitq.dat. A typical control file is given below-

$5.0 \mathrm{~d}-6,1.0 \mathrm{~d}-6$ starttime, timestep

$50.0 \mathrm{~d} 0,0.0 \mathrm{~d} 0$

$\mathrm{H}$ distance $\mathrm{V}$ distance between source and receiver

1

$0.0 \mathrm{~d} 0$

$0.314321 \mathrm{~d}-25$

$0.17445 \mathrm{~d}-21$

$0.62765 \mathrm{~d}-18$

$0.345474454392273496 \mathrm{~d}-7$

$1.05 \mathrm{~d} 0 \mathbf{0 . 0 9 5 \mathrm { d } 0} 0.818 \mathrm{do}$

regularizing parameter

' $a p h=0.7158769 \mathrm{~d}-7$, noi $=0.03, \mathrm{t}=(\mathrm{d}-5, \mathrm{~d}-3)$ '

$0.34454392273469 \mathrm{~d}-7$

regularizing parameter

The distances should be in meters and the times in seconds. Apart from the first two lines the only other parts of the control file the user needs to change are the starting conductivity (cond) value and the regularizing parameters. The starting conductivity value 
is the value from which the program begins to iterate. The closer it is to the actual value the quicker and more accurate the result is. Therefore, in cases where the conductivity is not known beforehand, if the conductivity obtained from the result differs greatly from that used in the control file the new value must be used in the control file and the program run again. Such an iterative procedure is necessary in order to achieve the greatest possible accuracy. The ideal value for the regularizing parameters is dependent on the noise in the data. Higher noise content would require increasing its value. The trade-off is that resolution would decrease.

\section{Running the program}

There are three programs trans.out, emtq.exe and qread that have to be run one after the other. In the directory that contains only one input file fort. 11 and its control file emtq.dat the program transp.out or transn.out has to be run depending on whether the initial part of the input data (the part before the zero-crossing) is positive or negative. It asks for the number of input data points (1024), output data points (400) and the start time. This program creates an output file fort.99. Then emtq.exe has to be run and it produces the output file fort.22 as well as screen output (output 1). In order to direct the screen output to a file the following command may be used -

emtq.exe $<$ emtq.dat $>$ \&out 1. dat \&

\section{Output}

Fort.22 contains the transformed waveform values for discrete steps of $\mathrm{q}$. The program qread changes the format of this file to one of single column which can be more easily handled by graphical package. Fort. 22 has ten different wavefield signals each calculated at a different start time of the time domain signal starting from the startime given in the control file with increments of one microsecond. The step size in the transformed domain is determined by the values in the control file. The step size is $d q=q$ step $\times \sqrt{\mu \times \text { cond }}$.

The screen output which is directed to outl.dat picks out the first arrivals in terms of $\mathrm{q}$ steps. 